\title{
Robust Permanent Income and Pricing
}

\author{
by \\ Lars Peter Hansen \\ University of Chicago, NBER and NORC \\ and \\ Thomas J. Sargent \\ Hoover Institution, Stanford University \\ University of Chicago \\ and \\ Thomas D. Tallarini, Jr. \\ Carnegie Mellon University
}

May 11, 1997

This research was funded by grants from the National Science Foundation. We thank Andrew Abel, Evan Anderson, John Cochrane, Cristina de Nardi, Martin Eichenbaum, John Heaton, Narayana Kocherlakota, Wen-Fang Liu, Jesus Santos, Kenneth Singleton and Nancy Stokey for useful criticisms of earlier drafts. We are grateful to Wen-Fang Liu for excellent research assistance. We thank two referees of an earlier draft for comments that prompted an extensive reorientation of our research. 


\section{Robust Permanent Income and Pricing}

"... I suppose there exists an extremely powerful, and, if I may so speak, malignant being, whose whole endeavors are directed toward deceiving me." Rene Descartes, Meditations, II. ${ }^{1}$

\section{Introduction}

This paper uses a permanent income model as a laboratory to study how consumption/savings profiles and security market prices are altered when consumers are 'robust decision makers'. Robust decision makers use the same probabilistic specification of the income and endowment shocks as would expected utility maximizers. But they regard this specification as a reference point about which they suspect small specification errors. They want decisions to be insensitive to these errors. This leads them to focus on consequences of their decisions under 'worst cases'. We show that such a preference for robustness lies concealed within the quantity implications of the permanent income model, and how its presence can be coaxed out of market-based measures of 'risk-aversion'. Indeed, we show that large market-based measures of risk aversion can emerge from a concern about making small specification errors.

The first part of this paper reinterprets the decision rules for saving and consumption from a rational expectations version of Hall's (1978) permanent income model. We verify identical behavior by someone who does not know enough about the probability laws for income and preference shocks to have rational expectations, and who manages his ignorance about them in a sophisticated and cautious way. ${ }^{2}$ We show that this new interpretation of Hall's decision rules serves to move the 'market price of risk' closer to empirical estimates.

Under a rational expectations interpretation, Hall's model excludes 'precautionary savings,' as emphasized by Zeldes (1989). Under our 'robust decision' interpretation, precautionary savings emerge out of the decision maker's 'worst case' analysis. Two offsetting changes leave the decision rules unchanged. One change is to impute 'irrational expectations' in the form of a particular class of misspecifications of the laws of motion of the exogenous income and preference shocks. By itself, this would increase the household's demand for capital, via a precautionary savings motive. We can offset that effect by changing

1 Descartes (1901, p. 227).

2 Our setting relates to the max-min utility theory of Gilboa and Schmeidler (1989) and Epstein and Wang (1994). A robust decision maker uses rules that work well for a specific stochastic environment, but that are also insensitive to small perturbations of the probabilistic specification (see Zames (1981), Frances (1987), and Zhou, Glover, and Doyle (1996)). Similarly, by ascribing a family of possible probability laws to a decision maker, the literature draws a sharp distinction between Knightian uncertainty and risk. Knightian uncertainty corresponds to the 'perturbations' in the probabilistic specification envisioned by the robust control theorists. 
the discount factor by enough to leave Hall's original decision rule intact. ${ }^{3}$ We base our estimation strategy on this observational equivalence result.

Actually, there is a third reinterpretation of Hall's decision rule, which emerges because in formulating possible specification errors, we build on a literature on risk-sensitive control started by Jacobson (1973, 1977), and extended by Whittle (1982, 1983, 1989, 1990) and others. This literature sought to enhance the impact of risk in control problems under what amount to 'rational expectations.' The literature induced bigger effects of risk on decision rules (i.e., greater departures from 'certainty equivalence') through a single additional 'risk sensitivity parameter' that alters the intertemporal objective of the decision-maker. For undiscounted linear-quadratic control problems, Glover and Doyle (1988) connected the risk-sensitive formulation to a type of robustness. They showed how the risk sensitivity parameter can be reinterpreted in terms of concern about particular types of misspecification. We use a discounted version of James's (1995) notion of robustness. Because we adopt a formulation of robust decision theory induced by the risk-sensitivity parameterization, we are free to regard the consumer in our economy as being risk sensitive and as forecasting the future using a correctly specified probability model (i.e., as having rational expectations). This leads to a third interpretation of the decision rules from Hall's model. We attain this reinterpretation by modeling risk sensitivity with discounting in a recursive manner, as in Epstein (1988), Weil (1989), Epstein and Zin (1989) and Hansen and Sargent (1995). ${ }^{4}$ Because of the triple interpretation of the decision rules, readers skeptical about robustness are free to read our paper from the vantage point of a rational expectations reformulation of the permanent income model in which preferences are recursive and risk sensitive. However, we prefer the robustness interpretation because it puts a new perspective on market based measures of risk aversion.

Although our reinterpretation of the permanent income model preserves decisions for consumption and investment, it moves asset prices. We investigate the asset pricing implications, and use them to help select a parameterization that matches some pricing patterns, while preserving the implications for saving and consumption. In contrast to models in the spirit of Bewley (1977), market incompleteness plays no role in our decentralization of the permanent income model. Instead, following Hansen (1987), we interpret the permanent income decision rule in terms of a social planning problem, where the consumption and investment processes are the equilibrium quantity allocations for a competitive equilibrium. We then deduce asset prices in the manner of Lucas (1978) and Epstein (1988), namely as shadow prices that clear security markets. These asset prices encode information about the

3 In effect, we are solving a particular 'robust control' version of an 'inverse optimal decision' problem. Versions of such problems have played an important role in the development of rational expectations theory. See Muth (1960). See Hansen and Sargent (1983) and Christiano (1987) for extensions of Muth's work.

4 Epstein and Zin (1989) developed a version of recursive utility theory that raises the market price of risk without altering the intertemporal substitution elasticity. Van Der Ploeg (1993) introduced risk sensitivity into a permanent income model, but not in a recursive manner. 
slopes of intertemporal indifference curves passing through the equilibrium consumption process, and therefore measure the risk aversion of the consumer. To accommodate robustness, our decentralization copies Epstein and Wang's (1994). We study how the asset pricing implications are altered by introducing a preference for robustness. In this way, we show how the consumer's preoccupation with mistakes gets transmitted into equilibrium security prices. ${ }^{5}$ We actually describe two asset pricing theories, one associated with the 'risk-sensitivity', another with the 'robust decision theory' interpretation of Hall's decision rule. Though the quantity implications of these reinterpretations of Hall's decision rule are identical, the asset pricing implications are slightly different. For our empirical application, the quantitative differences are small.

The remainder of this paper is organized as follows. Section 2 summarizes the necessary decision theory. We link risk-sensitive and robust decision theories by displaying two closely connected value functions associated with superficially different problems. The problems lead to identical decision rules. The second problem embodies a preference for robustness, provides links to Gilboa-Schmeidler's version of Knightian uncertainty, and explains the quote from Descartes. In sections 3 and 4, we describe and estimate our permanent income model. The observational equivalence proposition of section 4 motivates a two part strategy for using the quantity and asset price data. Section 5 exploits the links between robustness and risk-sensitivity in developing asset pricing formulas in terms of probability measures induced by 'pessimistic' views of laws of motion that emerge as by-products of robust decision making. These formulas prepare the way for our interpretations of the market price of risk in terms of robustness. Section 6 quantifies the amount of preference for robustness required to push up the market price of risk. Section 7 measures intertemporal mean-risk trade-offs associated with different amounts of concern with robustness. Section 8 concludes.

\section{Recursive Risk Sensitive Control}

The theory rests on two closely related recursive linear quadratic optimization problems. We describe a distortion of beliefs (i.e., a deviation from rational expectations) that induces the same behavior as a particular modification of preferences toward risk. The equivalence of these two problems lets us interpret a 'risk sensitivity' parameter as measuring a preference for robustness.

The recursive risk sensitive control problem

The state transition equation is

$$
x_{t+1}=A x_{t}+B i_{t}+C w_{t+1} .
$$

\footnotetext{
5 See Melino and Epstein (1995) for an alternative attack on this same question. They use a recursive formulation of an $\epsilon$-contamination specification adapted from the theory of robust statistics.
} 
where $i_{t}$ is a control vector, $x_{t}$ is the state vector, and $w_{t+1}$ is an i.i.d. Gaussian random vector with $E w_{t+1}=0$, and $E w_{t+1} w_{t+1}^{\prime}=I$. Let $J_{t}$ be the sigma algebra induced by $\left\{x_{0}, w_{s}, 0 \leq s \leq t\right\}$. The one-period return function is

$$
u(i, x)=-i^{\prime} Q i-x^{\prime} R x
$$

where $Q$ is positive definite and $R$ is positive semidefinite. Following Epstein and Zin (1989), Weil (1993), and Hansen and Sargent (1995), we use the following recursion to induce intertemporal preferences:

$$
U_{t}=u\left(i_{t}, x_{t}\right)+\beta \mathcal{R}_{t}\left(U_{t+1}\right)
$$

where

$$
\mathcal{R}_{t}\left(U_{t+1}\right) \equiv \frac{2}{\sigma} \log E\left[\exp \left(\frac{\sigma U_{t+1}}{2}\right) \mid J_{t}\right] .
$$

When $\sigma=0$ we take $\mathcal{R}_{t} \equiv E\left(U_{t+1} \mid J_{t}\right)$, and we have the usual von Neumann-Morgenstern form of state additivity. When $\sigma \neq 0$, the operator $\mathcal{R}_{t}$ makes an additional risk adjustment over and above that induced by the shape of $u(\cdot, \cdot)$. Values of $\sigma$ less than zero correspond to more aversion to risk vis a vis the von Neumann-Morgenstern specification. ${ }^{6}$ As emphasized by Hansen and Sargent (1995a), the (log, exp) specification links the general recursive utility specification of Epstein and Zin (1989) to risk-sensitive control theory. The same (log, exp) specification was used in Weil's (1993) version of the permanent income model, but he did not exploit connections to the risk-sensitive control literature.

The risk sensitive control problem is to maximize the time zero utility index $U_{0}$ by choosing a control process $i_{t}$ adapted to $J_{t}$. Let $W(x)$ denote the optimum value function for this problem, so that $W\left(x_{0}\right)=U_{0}^{e}$. Hansen and Sargent (1995) extended the JacobsonWhittle risk-sensitive control theory to provide formulas for $\Omega$ and $\rho$ in the following representation of the value function:

$$
U_{t}^{e}=W\left(x_{t}\right)=x_{t}^{\prime} \Omega x_{t}+\rho .
$$

Let $i=-F x$ denote the optimal decision rule.

We shall have cause to evaluate $\mathcal{R}_{t}\left(U_{t+1}\right)$ for the quadratic value function $(3)$ where $\Omega$ is a negative semidefinite matrix of real numbers and $\rho$ is a nonpositive real number. It follows from Jacobson (1973), that

$$
\mathcal{R}_{t}\left(U_{t+1}^{e}\right)=x_{t}^{\prime} \hat{\Omega} x_{t}+\hat{\rho}
$$

6 As in Kreps and Porteus (1978), this recursive utility formulation overturns the indifference to the timing of the resolution of uncertainty inherent in state-separable preferences. The additional risk adjustment for $\sigma<0$ implies a preference for early resolution of uncertainty. 
where

$$
\hat{\Omega}=A^{\prime}\left[\Omega+\sigma \Omega C\left(I-\sigma C^{\prime} \Omega C\right)^{-1} C^{\prime} \Omega\right] A
$$

and

$$
\hat{\rho}=\rho-(1 / \sigma) \log \left[\operatorname{det}\left(I-\sigma C^{\prime} \Omega C\right)\right]
$$

so long as the matrix $\left(I-\sigma C^{\prime} \Omega C\right.$ ) is positive definite, which we assume.

\section{A related game}

The Markov perfect equilibrium of a particular two player game can be represented in terms of a single value function $\tilde{W}(x)$ that is related to $W(x)$. This is a special game in which it turns out that sequential play, under the Markov perfect equilibrium concept, leads to the same outcome as the game where players can precommit at time 0 . Therefore, as in a single-agent control problem, we are free to pose and solve the problem recursively, or once-and-for-all from the vantage point of an initial period. The game has one player choosing contingency rules for the control vector $\left\{i_{t}\right\}$, with two differences vis a vis the single agent risk-sensitive control problem. First, this agent does not make a risk adjustment in the utility function; and second, a distortion is introduced every time period into the conditional mean of the shock process. Thus, one player maximizes a utility index $\tilde{U}_{0}=E_{0} \sum_{t=0}^{\infty} \beta^{t} u\left(i_{t}, x_{t}\right)$ by choice of state-feedback rules for $\left\{i_{t}\right\}$ and subject to the distorted law of motion

$$
x_{t+1}=A x_{t}+B i_{t}+C\left(w_{t+1}+v_{t}\right),
$$

where $v_{t}$ acts as a distortion of the mean of the innovation. A second player is introduced as a device for determining the conditional mean distortions $\left\{v_{t}\right\}$ in a way that delivers a specific form of robustness. We want the feedback rule for $i_{t}$ to be insensitive to mistakes $v_{t}$ in the conditional mean of $w_{t+1}$. To attain this insensitivity, we have the second player be a malevolent opponent that minimizes $\tilde{U}_{0}$ over state feedback rules for $v_{t}$. This opponent's choice of rule for $v_{t}$ is restrained by

$$
E_{0} \sum_{t=0}^{\infty} \beta^{t}\left|v_{t} \cdot v_{t}\right| \leq \eta_{0} .
$$

The Markov perfect equilibrium of this game has a value function that satisfies:

$$
\begin{aligned}
\tilde{W}(x) & =\inf _{v} \sup _{i}\left\{-i^{\prime} Q i-x^{\prime} R x+\beta\left[-\frac{1}{\sigma} v^{\prime} v+E \tilde{W}(A x+B i+C(w+v))\right]\right\} \\
& =x^{\prime} \Omega x+\tilde{\rho}
\end{aligned}
$$


where the $E$ operator integrates $w$ with respect to a normal distribution with mean zero and covariance matrix $I$. Notice that this two-player min-max game can be characterized in terms of a single value function $\tilde{W}$ and so can be viewed as a zero sum game. Hansen and Sargent (1997) show that the value functions $W$ and $\tilde{W}$ share the same matrix $\Omega$ in their quadratic forms, but have different constants $\rho$ and $\tilde{\rho}$. Let $i=-F x, v=G x$ denote the policy rules that solve (8); the rules are linear, and the same rule for $i$ also solves the risk-sensitive control problem.

The relationship between the two value functions and the decision rules for $i$ establishes how the risk-sensitive preference specification induces the same behavior that would occur without the risk-sensitivity adjustment to preferences, but with the pessimistic view of the conditional mean of innovations (the $v_{t}$ 's) reflected in (8). In this 'pessimistic' formulation, $-\sigma^{-1}$ in (8) becomes a Lagrange multiplier on restriction (7). Here $\eta_{0}$ indexes the degree of pessimism, i.e., the size of the domain of sequences from which the 'malevolent opponent' selects adverse $v_{t}$ 's. Hansen and Sargent (1997) describe in detail why it is convenient computationally to parameterize pessimism in this way.

\section{'Uncertainty aversion' or robustness}

The Markov perfect equilibrium summarized by (8) is the value function for a single decision maker whose decisions are governed by a 'worst case' analysis. By using a feedback rule of $i_{t}$ that solves (8), the robust controller does better for some appropriately constrained mistake sequences $\left\{v_{t}\right\}$ while sacrificing utility when these mistakes are precluded. Our treatment of this robustness and its connection to risk sensitivity follows closely James's (1995) recent survey of robust control, except that we have incorporated discounting into the risk sensitive formulation of the problem and into the corresponding constraints on the model misspecification.

There is a closely related literature in economics spawned by the work of Gilboa and Schmeidler (1989) and Epstein and Wang (1994). The decision theory axiomatized by Gilboa and Schmeidler generalizes expected utility theory by studying a setting where decisions are based on a 'maxmin' criterion because beliefs are described by a family of probability measures rather than a single probability measure. In our setup, there is a 'nominal model' corresponding to setting $v_{t}=0$ for all $t$. Alternative specification error sequences $\left\{v_{t}\right\}$ constrained by (7) deliver the resulting family of stochastic processes used in the state evolution equation. Hence our decision maker can be viewed as being endowed with preferences represented by the maxmin utility theory of Gilboa and Schmeidler. Following Epstein and Wang (1994), we can interpret the nonuniqueness of the stochastic constraints as depicting a form of Knightian uncertainty: an ambiguity of beliefs not fully specified in probabilistic terms. This ambiguity is captured by our constrained sequence of specification errors obeying (7).

In intertemporal contexts, Epstein and Wang (1994) use a Markov formulation of the two-player game to avoid inducing a form of time inconsistency. We follow the literature 
on robust control by holding fixed the Lagrange multiplier $-\sigma^{-1}$ on the specification error constraint over time. This requires imposing the following sequence of restrictions on the 'malevolent opponent':

$$
E_{t} \sum_{j=0}^{\infty} \beta^{j}\left|v_{t+j} \cdot v_{t+j}\right| \leq \eta_{t},
$$

where $\eta_{t}$ is a 'continuation pessimism bound.' 7 Below, we shall compute the left side of (9), and use it to measure the amount of uncertainty aversion associated with alternative values of $\sigma$. To compute this measure, we avail ourselves of a formula for the matrix $G$ in $v=G x$.

\section{Solution for $v$}

The solution for $v$ within the Markov perfect equilibrium satisfies:

$$
\hat{v}_{t}=\sigma\left(I-\sigma C^{\prime} \Omega C\right)^{-1} C^{\prime} \Omega A^{o} x_{t},
$$

where $x_{t+1}=A^{o} x_{t}+C w_{t+1}$ under the optimal control law for the risk-sensitive problem. (Here we are assuming that the parameter $\sigma$ is sufficiently small that the matrix ( $I-$ $\sigma C^{\prime} \Omega C$ ) is positive definite. $)^{8}$

Below we shall compute $\hat{v}_{t}$ and study how it alters measures of risk aversion extracted from asset prices.

\section{Modified certainty equivalence}

Whittle (1981) pointed out how the solution for $v$ supports a modified version of certainty equivalence. This version asserts the equivalence of two ways of evaluating timeinvariant decision rules $i_{t}=-F x_{t}$, one under rational expectations and risk-sensitive preferences; the other under distorted expectations and ordinary $(\sigma=0)$ quadratic preferences. Let $A^{*}=(A-B F), R^{*}=R+F^{\prime} Q F$. The two valuation algorithms are:

1. $U_{t}^{e}=-x_{t}^{\prime} R^{*} x_{t}+\beta \mathcal{R}_{t} U_{t+1}^{e}$, where $\mathcal{R}_{t}$ is defined in (2), and where the conditional expectation operator in (2) is computed with respect to the (true) law of motion $x_{t+1}=$ $A^{*} x_{t}+C w_{t+1}$. The criterion can be represented as the translated quadratic form

\footnotetext{
7 Hansen and Sargent (1997) discuss how the particular parameterization of 'uncertainty aversion' embedded in (8) - in which the 'Lagrange multiplier' $-\sigma^{-1}$ is time invariant - requires choosing the continuation pessimism bounds $\eta_{t}$ in a way to make the opponent's decision problem recursive.

8 Although the matrix $\Omega$ depends implicitly on $\sigma$, it can be shown that the requisite positive definiteness will be satisfied for small values of $\sigma$. The risk-sensitive control theory literature draws attention to the breakdown point under which this positive definiteness property ceases to hold (e.g., see Glover and Doyle (1988)). At such points, the risk-adjusted recursive utility is $-\infty$ regardless of the controller's action. The general equilibrium aspects of our analysis lead us to look at much smaller risk corrections than are tolerated by the breakdown analysis.
} 
$U_{t}^{e}=x_{t}^{\prime} \Omega x_{t}+\rho$, where the matrix $\Omega$ and the scalar $\rho$ are fixed points of operators defined by Hansen and Sargent (1995a).

2. $\tilde{U}_{t}=-x_{t}^{\prime} R^{*} x_{t}+\beta \tilde{E}_{t} \tilde{U}_{t+1}$, where $\tilde{E}_{t}$ is an expectation operator computed with respect to the distorted law of motion

$$
x_{t+1}=\hat{A} x_{t}+C w_{t+1}
$$

where

$$
\hat{A}=\left[I+\sigma C\left(I-\sigma C^{\prime} \Omega C\right)^{-1} C^{\prime} \Omega\right] A^{*} .
$$

The formula for $\hat{A}$ is derived by adding $C \hat{v}_{t}$ to $A^{*}$, where $\hat{v}_{t}$ satisfies a version of (10) with $A^{o}=A^{*}$. The criterion $\tilde{U}_{t}$ has the representation $\tilde{U}_{t}=x_{t}^{\prime} \Omega x_{t}+\tilde{\rho}$, where $\Omega$ is the same matrix occurring in the first representation.

Evidently, these two evaluations yield the same ordering over time-invariant decision rules $i_{t}=-F x_{t}$. This is the modified certainty equivalence principle. Notice the appearance of $\Omega$, computed from the first formulation, in the construction of the distorted law of motion (12). We shall use $\hat{A}$ from (12) again in computing asset prices.

\section{Robust Permanent Income Theory}

Hall (1978), Campbell (1987), Heaton (1993), and Hansen, Roberds, and Sargent (1991) studied how closely a permanent income model approximates aggregate data on consumption and investment. We formulate a risk-sensitive version of the permanent income model with habit persistence, estimate it from data on consumption and investment, then use it to compare the implications of risk-sensitivity for consumption and investment, on the one hand, and asset prices, on the other. We demonstrate an observational equivalence proposition asserting that the consumption and investment data alone are insufficient simultaneously to identify the risk-sensitivity parameter $\sigma$ and the subjective discount factor $\beta$. This observational equivalence substantiates our claim to be reinterpreting decision rules from a habit-persistence version of Hall's model in terms of robust decision making. Furthermore, adding knowledge of the risk-free rate, which is constant in this model, does not achieve identification. But later we will show that the risk-sensitivity parameter has strong effects on other asset prices, including the market price of risk.

The lack of identification from consumption and investment data emerges as follows. For a given specification of shocks, introducing risk sensitivity provides an additional precautionary motive for saving. In terms of implications for savings, this motive can be offset by diminishing the subjective discount factor to make saving less attractive. In terms of effects on the valuation of risky assets, these changes are not offsetting. 


\section{The model}

We formulate the model in terms of a planner with preferences over consumption streams $\left\{c_{t}\right\}_{t=0}^{\infty}$, intermediated through the service stream $\left\{s_{t}\right\}$. Preferences are ordered by the utility index $U_{0}$, defined through the recursion

$$
U_{t}=-.5\left(s_{t}-b_{t}\right)^{2}+\beta \mathcal{R}_{t}\left(U_{t+1}\right)
$$

where $\mathcal{R}_{t}\left(U_{t+1}\right)$ is defined by (2).

In (13), $s_{t}$ is a scalar household service produced by the scalar consumption $c_{t}$ via the household technology

$$
\begin{aligned}
& s_{t}=(1+\lambda) c_{t}-\lambda h_{t-1}, \\
& h_{t}=\delta_{h} h_{t-1}+\left(1-\delta_{h}\right) c_{t}
\end{aligned}
$$

where $\lambda>0$ and $\delta_{h} \in(0,1)$. In (13), $\left\{b_{t}\right\}$ is an exogenous preference shock process. System (14) accommodates habit persistence or rational addiction as in Ryder and Heal (1973), Becker and Murphy (1988), Sundaresan (1989), Constantinides (1990) and Heaton (1993). By construction, $h_{t}$ is a geometric weighted average of current and past consumption. Setting $\lambda>0$ induces intertemporal complementarities. Consumption services depend positively on current consumption, but negatively on a weighted average of past consumptions, an embodiment of 'habit persistence'.

There is a linear production technology

$$
c_{t}+i_{t}=\gamma k_{t-1}+d_{t}
$$

where the capital stock $k_{t}$ at the end of period $t$ evolves according to

$$
k_{t}=\delta_{k} k_{t-1}+i_{t},
$$

$i_{t}$ is time $t$ gross investment, and $\left\{d_{t}\right\}$ is an exogenously specified endowment process. The parameter $\gamma$ is the (constant) marginal product of capital, and $\delta_{k}$ is the depreciation factor for capital. Solving the capital evolution equation for investment and substituting into the linear production technology gives:

$$
c_{t}+k_{t}=\left(\delta_{k}+\gamma\right) k_{t-1}+d_{t}
$$

We define:

$$
R \equiv \delta_{k}+\gamma
$$

which is the physical (gross) return on capital taking account the fact that capital depreciates over time. When the economy is decentralized, $R$ will also coincide with the gross 
return on a risk free asset. We impose that the solution for $\left\{c_{t}, h_{t-1}, k_{t-1}\right\}$ belong to $L_{0}^{2}$, the space of stochastic processes $\left\{y_{t}\right\}$ defined as:

$$
L_{0}^{2}=\left\{y: y_{t} \text { is in } J_{t} \text { for } t=0,1, \cdots \text { and } E \sum_{t=0}^{\infty} R^{-t} y_{t} \cdot y_{t} \mid J_{0}<+\infty\right\}
$$

We suppose that the endowment and preference shocks $\left(d_{t}, b_{t}\right)$ are governed by $b_{t}=$ $U_{b} z_{t}, d_{t}=U_{d} z_{t}$ where

$$
z_{t+1}=A_{22} z_{t}+C_{2} w_{t+1}
$$

Here $w_{t+1}$ is independent of $J_{t}=\left\{w_{t}, w_{t-1}, \ldots, w_{1}, z_{0}\right\}$, the eigenvalues of $A_{22}$ are bounded in modulus by unity, and $w_{t+1}$ is normally distributed with mean zero and covariance matrix $I$.

Given $k_{0}$, the planner chooses a sequence $\left\{c_{t}, k_{t}\right\}$ in $L_{0}^{2}$ to maximize $U_{0}$ subject to (14), (15). ${ }^{9}$

\section{Solution of model and identification of $\sigma$}

To establish observational equivalence for the quantity observations, we proceed constructively. First, we compute a solution for $\sigma=0$ and $\beta R=1$, i.e., a permanent income economy without risk sensitivity. Then we use the allocation for this $\sigma=0$ economy to construct an equivalence class of alternative $(\sigma, \beta)$ 's that generate the same allocation, for fixed values of all the other parameters. This demonstrates that the pair $(\sigma, \beta)$ is not identified from quantity observations alone.

The $\sigma=0, \beta R=1$ benchmark case

To produce a permanent income model in the $\sigma=0$ special case, we follow Hall (1978) and impose that $\beta R=1$. When $\sigma=0,(13)-(2)$ reduces to

$$
U_{0}=E_{0} \sum_{t=0}^{\infty} \beta^{t}\left\{-.5\left(s_{t}-b_{t}\right)^{2}\right\}
$$

Formulate the planning problem as a Lagrangian by putting random Lagrange multiplier processes of $\beta^{t} \mu_{s t}$ on (14a), $\beta^{t} \mu_{h t}$ on (14b), and $\beta^{t} \mu_{c t}$ on (15). First-order necessary

\footnotetext{
9 We can convert this problem into a special case of the control problem posed in section 2 as follows. Form a composite state vector $x_{t}$ by stacking $h_{t_{1}}, k_{t-1}$ and $z_{t}$, let the control $i_{t}$ be given by $s_{t}-b_{t}$. Solve (14a) for $c_{t}$ as a function of $s_{t}-b_{t}, b_{t}$ and $h_{t-1}$ and substitute into equations (14b) and (15). Stack the resulting two equations along with the state evolution equation for $z_{t}$ to form the evolution equation for $x_{t+1}$.
} 
conditions are

$$
\begin{aligned}
\mu_{s t} & =b_{t}-s_{t} \\
\mu_{c t} & =(1+\lambda) \mu_{s t}+\left(1-\delta_{h}\right) \mu_{h t} \\
\mu_{h t} & =\beta E_{t}\left[\delta_{h} \mu_{h t+1}-\lambda \mu_{s t+1}\right] \\
\mu_{c t} & =\beta R E_{t} \mu_{c t+1},
\end{aligned}
$$

and also (14), (15). When $\beta R=1$, equation (17d) implies that $\mu_{c t}$ is a martingale; then (17b) and (17c) solved forward imply that $\mu_{s t}, \mu_{h t}$ are also martingales. This implies that $\mu_{\text {st }}$ has the representation

$$
\mu_{s t}=\mu_{s t-1}+\nu^{\prime} w_{t}
$$

for some vector $\nu$.

Use (17a) to write $s_{t}=b_{t}-\mu_{s t}$, substitute this into the household technology (14), and rearrange to get the system

$$
\begin{aligned}
c_{t} & =\frac{1}{1+\lambda}\left(b_{t}-\mu_{s t}\right)+\frac{\lambda}{1+\lambda} h_{t-1} \\
h_{t} & =\tilde{\delta}_{h} h_{t-1}+\left(1-\tilde{\delta}_{h}\right)\left(b_{t}-\mu_{s t}\right)
\end{aligned}
$$

where $\tilde{\delta}_{h}=\frac{\delta_{h}+\lambda}{1+\lambda}$. Equation (19b) can be used to compute

$$
E_{t} \sum_{j=0}^{\infty} \beta^{j} h_{t+j-1}=\left(1-\beta \tilde{\delta}_{h}\right)^{-1} h_{t-1}+\frac{\beta\left(1-\tilde{\delta}_{h}\right)}{\left(1-\beta \tilde{\delta}_{h}\right)} E_{t} \sum_{j=0}^{\infty} \beta^{j}\left(b_{t+j}-\mu_{s t+j}\right) .
$$

For the purpose of solving the first-order conditions (17), (14), (15) subject to the side condition that $\left\{c_{t}, k_{t}\right\} \in L_{0}^{2}$, treat the technology (15) as a difference equation in $\left\{k_{t}\right\}$, solve forward, and take conditional expectations on both sides to get

$$
k_{t-1}=\sum_{j=0}^{\infty} R^{-(j+1)} E_{t}\left(c_{t+j}-d_{t+j}\right)
$$

Use (19a) to eliminate $\left\{c_{t+j}\right\}$ from (21), then use (18) and (20). Solve the resulting system for $\mu_{s t}$, to get

$$
\mu_{s t}=\left(1-R^{-1}\right)^{-1} \sum_{j=0}^{\infty} R^{-j} E_{t} b_{t+j}+\psi_{0} \sum_{j=0}^{\infty} R^{-j} E_{t} d_{t+j}+\psi_{1} h_{t-1}+\psi_{2} k_{t-1},
$$

where $\psi_{0}, \psi_{1}, \psi_{2}$ are constants. Equations (22), (19), and (15) represent the solution of the planning problem. 
Notice that (22) makes $\mu_{s t}$ depend on a geometric average of current and future values of $b_{t}$. Therefore, both the optimal consumption service process and optimal consumption depend on the difference between $b_{t}$ and a geometric average of current and expected future values of $b$. So there is no 'level effect' of the preference shock on the optimal decision rules for consumption and investment. However, the level of $b_{t}$ will affect equilibrium asset prices.

Observational equivalence (for quantities) of $\sigma=0$ and $\sigma \neq 0$

At this point, we state the following

Observational Equivalence Proposition: Given $\beta R=1$, the optimal consumptioninvestment plan without risk-sensitivity $(\sigma=0)$ is identical to the optimal risk-sensitive $(\sigma<0)$ plan, but for a smaller discount factor $\hat{\beta}$.

This proposition means that, so far as the quantities $\left\{c_{t}, k_{t}\right\}$ are concerned, the risksensitive $(\sigma<0)$ version of the permanent income model is observationally equivalent to the benchmark $(\sigma=0)$ version. This insight will guide our estimation strategy, because it sharply partitions the impact of risk-sensitivity into real and pricing parts.

The proof of the proposition is by construction.

Proof: This is the plan of the proof. Begin with a solution $\left\{\bar{s}_{t}, \bar{c}_{t}, \bar{k}_{t}, \bar{h}_{t}\right\}$ for a benchmark $\sigma=0$ economy. Form a comparison economy with a $\sigma \in[\underline{\sigma}, 0]$, where $\underline{\sigma}$ is the boundary of an admissible set of $\sigma$ 's to be described below. Fix all parameters except $(\sigma, \beta)$ the same as in the benchmark economy. Conjecture that $\left\{\bar{s}_{t}, \bar{c}_{t}, \bar{k}_{t}, \bar{h}_{t}\right\}$ is also the optimal allocation for the $\sigma<0$ economy. Finally, construct a $\beta=\hat{\beta}$ that verifies this conjecture.

Here are the details of the construction. The optimality of the allocation implies that $E_{t} \mu_{c t+1}=\mu_{c t}$, and that (18) and (22) are satisfied for the $\left({ }^{-}\right)$allocation, where $E_{t}$ is the expectation operator under the correct probability measure. The key idea is to form the distorted expectation operator $\hat{E}_{t}$, then choose $\beta=\hat{\beta}$ to make the distorted version of the Euler equation for $\mu_{c t}$ hold at the benchmark $(\sigma=0)$ allocation.

To compute the distorted expectation operator, we follow the recipe given in formulas (8), (12). First, we have to evaluate the utility index $U_{0}$ by using (8). We want to evaluate (13) with $s_{t}-b_{t} \equiv-\mu_{s t}$ and $\mu_{s t}$ given by the law of motion (18), which we take as exogenous because the allocation is frozen. We take $\mu_{s t}$ as the state. Since there is no control, (8) collapses to

$$
\Omega x^{2}=-x^{2}+\beta \min _{v}\left(-\frac{1}{\sigma} v^{2}+\Omega(x+\theta v)^{2}\right),
$$

and we write $\mu_{s t}=\mu_{s t-1}+\theta(v+w)$, where $\theta^{2}=\nu^{\prime} \nu$ and $v$ is the specification error 
'chosen' by the 'opponent' in the fictitious game. The scalar $\Omega$ that solves (23) is

$$
\Omega(\beta)=\frac{\beta-1+\sigma \theta^{2}+\sqrt{\left(\beta-1+\sigma \theta^{2}\right)^{2}+4 \sigma \theta^{2}}}{-2 \sigma \theta^{2}} .
$$

It follows from (12) that the distorted law of motion for $\mu_{s t}$ is

$$
E_{t} \mu_{s t+1}=\hat{\zeta} \mu_{s t}
$$

where

$$
\hat{\zeta}=\hat{\zeta}(\beta)=1+\frac{\theta^{2} \sigma \Omega(\beta)}{1-\sigma \theta^{2} \Omega(\beta)} .
$$

Since $\mu_{c t}$ is proportional to $\mu_{s t}$, it follows that

$$
E_{t} \mu_{c t+1}=\hat{\zeta} \mu_{c t}
$$

with the same $\hat{\zeta}$ given by (26). In terms of the distorted expectation operator, the Euler equation for capital is

$$
\hat{\beta} R E_{t} \mu_{c t+1}=\mu_{c t}
$$

or

$$
\hat{\beta} R \hat{\zeta}(\hat{\beta})=1 \text {. }
$$

Let $\underline{\sigma}$ be the lowest value for which the solution of (24) is real. Then given $\sigma \in(\underline{\sigma}, 0]$, there exists a $\hat{\beta}$ satisfying (28) such that for $(\sigma, \hat{\beta})$ the benchmark allocation solves the risk-adjusted problem. Therefore equations $(24),(26)$, and (28) define a locus of ( $\sigma, \hat{\beta})$ 's, each point of which is observationally equivalent to $(0, \beta)$ for $\left(c_{t}, k_{t}\right)$ observations, because each supports the benchmark allocation.

Furthermore, according to the asset pricing theory to be developed shortly and (28), the price of a sure claim on consumption one period ahead is $R^{-1}$ for all $t$ and all $(\sigma, \hat{\beta})$ in the locus. Therefore, these different parameter pairs are also observationally equivalent with respect to the risk-free rate. ${ }^{10}$

In Figure 1, we report the $(\sigma, \beta)$ pairs that are observationally equivalent for our maximum likelihood estimates for the remaining parameters, which we are about to describe.

10 In this model, the technology (15) ties down the risk-free rate. For a version of the model with quadratic costs of adjusting capital, the risk-free rate comes to depend on $\sigma$, even though the observations on quantities are nearly independent of $\sigma$. See Hansen and Sargent (1996). 


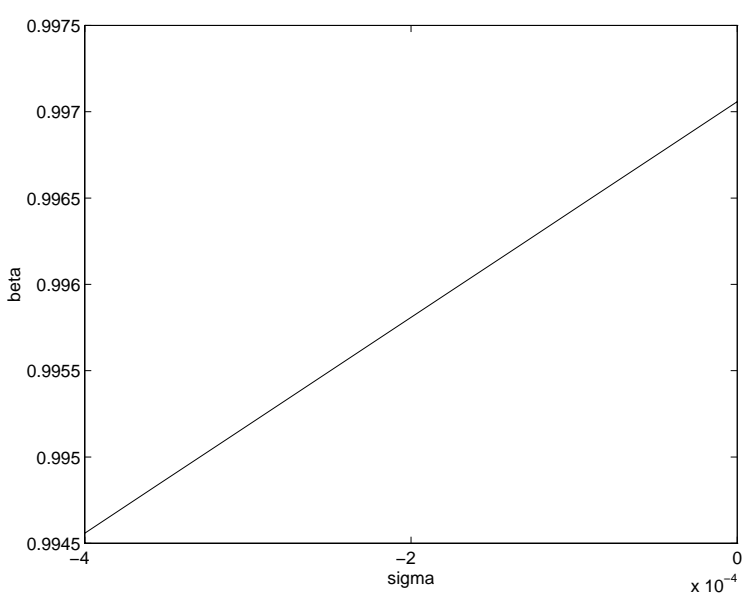

Figure 1. Observationally equivalent $(\sigma, \beta)$ pairs for maximum likelihood values of identified parameters; $\sigma$ is the ordinate, $\beta$ the coordinate.

The observational equivalence depicted in Figure 1 shows that by lowering the discount factor, we offset the precautionary savings motive by making investment in a perfect foresight world unattractive. As an indication of the important precautionary role for savings in this model, suppose that future endowments and preference shifters could be forecast perfectly. Then consumers would choose to draw down their capital stock. Investment would be sufficiently unattractive that the optimal linear rule would eventually have both consumption and capital cross zero. ${ }^{11,12}$ Thus our robust control interpretation of the permanent income decision rule, delivers a form of precautionary savings absent under the usual interpretation.

For any given pair $(\sigma, \beta)$ depicted in Figure 1 , the permanent income decision rule can viewed as reflecting either risk sensitivity or a concern for robustness. The familiar version of the precautionary savings motive focuses on the role of variation in the shocks. This version is delivered in our setup by the risk sensitive decision theoretic formulation.

\footnotetext{
11 Introducing nonnegativity constraints in capital and/or consumption would induce nonlinearities into the consumption and and savings rules, especially near zero capital. But investment would remain unattractive in the presence of those constraints for experiments like the one we are describing here. See Deaton (1991) for a survey and quantitative assessment of consumption models with binding borrowing constraints.

12 As emphasized by Carroll (1992), even when the discount factor is small are relative to the interest rate, precautionary savings can emerge when there is a severe utility cost for zero consumption. Such a utility cost is absent in our formulation.
} 
In contrast, the precautionary notion delivered by robust control theory emerges because consumers guards against mistakes in conditional means of shocks. Thus, the emphasis shifts from second to first moment properties of shocks. properties.

\section{Estimation}

Different observationally equivalent $(\sigma, \beta)$ pairs identified by our Proposition bear different implications about (i) the pricing risky assets; (ii) the amounts required to compensate the social planner for confronting different amounts of risk; (iii) the amount of model misspecification used to justify the planner's decisions if risk sensitivity is reinterpreted as aversion to Knightian uncertainty. To evaluate these implications, we first choose parameters, including noise variances, by estimating a $\sigma=0$ version of our permanent income model, conditioning the likelihood function only on U.S. post-war quarterly consumption and investment data. We estimated the permanent-income model with habit persistence using U.S. quarterly data on consumption and investment for the period 1970I-1996III. ${ }^{13}$

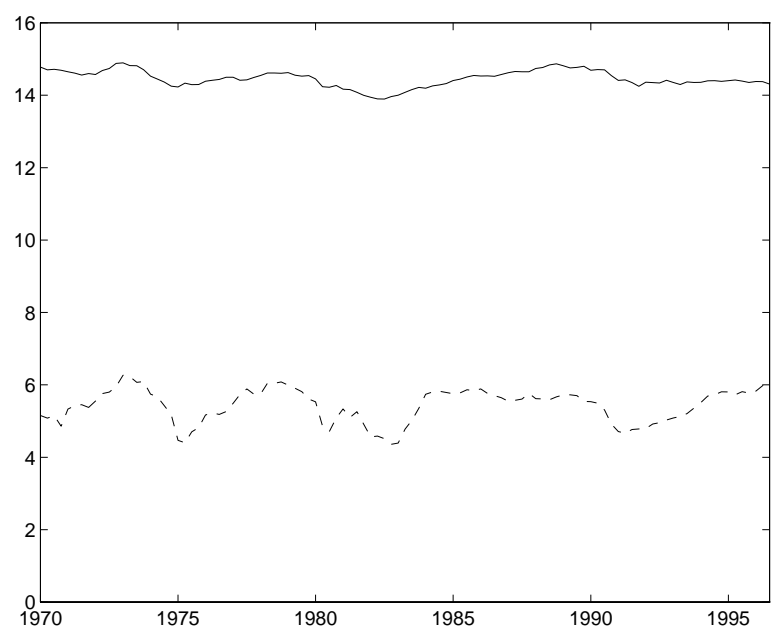

Figure 2. Detrended consumption and investment (dotted line) data.

\footnotetext{
13 Our choice of starting the sample in 1970 corresponds to the second subsample analyzed by Burnside, Eichenbaum and Rebello (1990). Thus we have omitted the earlier period of 'higher productivity' in our analysis. We initially estimated the model for the entire post war time period, but we found that the "productivity slowdown' was captured in our likelihood estimation by an initial slow decline in the preference shock process followed by a slow increase. Our illustrative permanent income model is apparently not well suited to capture productivity slowdowns. Given the empirical results reported in Burnside, Eichenbaum and Rebello (1990), the same could be said of the commonly used stochastic specification of Solow's growth model.
} 
Consumption is measured by nondurables plus services, while investment is measured by the sum of durable consumption and gross private investment. ${ }^{14}$ We applied the model to data that have been scaled through multiplication by $1.0033^{-t}$. The scaled time series are plotted in Figure 2. We estimated the model from data on $\left(c_{t}, i_{t}\right)$, setting $\sigma=0$, then deduced pairs $(\sigma, \beta)$ that are observationally equivalent. We estimated parameters by climbing a Gaussian likelihood function. We formed the likelihood function recursively, and estimated the unobserved part of the initial state vector using procedures described by Hansen and Sargent (1996).

We specified two shock processes: an endowment process $\left\{d_{t}\right\}$ and a preference shift process $\left\{b_{t}\right\}$. Because we are modeling two observed time series as functions of two shock processes, the model would lose its content were we to permit arbitrary cross correlation between the endowment and preference shock processes. Therefore, we assumed that these processes are orthogonal. In our initial estimation, we found that the preference shock process was particularly persistent. As a consequence, for the results presented here, we imposed a unit root and assumed that the first difference of the preference shock is a firstorder autoregression. For the endowment processes, we experimented with autorgressive processes of order 1,2 , and 3 , which revealed the log likelihood values depicted in Table 1. In the table, 'AR1' denotes the first-order autoregression, and so on. The likelihood values show a substantial gain in increasing the order from 1 to 2 , but little gain in going from 2 to 3 . These results led us to specify a second order autoregression for the endowment process.

\section{Table 1 \\ Likelihood Values}

endowment $2 \times$ LogLikelihood

specification

AR1

735.16

AR2

755.24

AR3

755.26

Note: The values reported differ from twice the log likelihood by a common constant.

We write the forcing processes as:

$$
\begin{gathered}
b_{t}^{*}-b_{t-1}^{*}=\alpha\left(b_{t-1}^{*}-b_{t-2}^{*}\right)+c_{b} w_{t}^{b} \\
d_{t}^{*}=\phi_{1} d_{t-1}^{*}+\phi_{2} d_{t-2}^{*}+c_{d} w_{t}^{d} .
\end{gathered}
$$

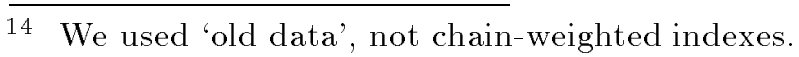


with

$$
b_{t}=b_{t}^{*}+\mu_{b}, d_{t}=d_{t}^{*}+\mu_{d} .
$$

Thus the forcing processes are governed by seven free parameters: $\left(\alpha, c_{b}, \phi_{1}, \phi_{2}, c_{d}, \mu_{b}, \mu_{d}\right)$. While the parameter $\mu_{b}$ alters the marginal utilities, as we noted previously, it does not influence the decision rules for consumption and investment. Consequently, we fixed $\mu_{b}$ at an arbitrary number, namely 32 , in our estimation.

The four parameters governing the endogenous dynamics are: $\left(\gamma, \delta_{h}, \beta, \lambda\right)$. We set $\delta_{k}=.975$. We initially did not impose the permanent income restriction, $\beta R=1$, but the restriction was satisfied by our estimates, so we proceeded to impose it. That is, our estimates confirmed the random walk prediction for both the marginal utility process for consumption goods and the marginal utility process for consumption services. The restrictions that $\beta R=1, \delta_{k}=.975$ pin down $\gamma$ once $\beta$ is estimated. We chose to impose a $\beta=.9971$, which after adjustment for the effects of the geometric growth factor of 1.0033 implies an annual real interest rate of $2.5 \% .{ }^{15}$

When estimated freely from consumption and investment data, the point estimate was considerably higher. However, imposition of this restriction caused only a modest reduction in the likelihood function.

In Table 2 we report our estimates for the parameters governing the endogenous and exogenous dynamics. In Figure 3 we report impulse response functions for consumption and investment to innovations in both the endowment and the preference shift processes. For sake of comparison, we also report estimates from a no habit persistence $(\lambda=0)$ model in Table 2, and the resulting impulse response functions in Figure 4.

Notice that the endowment shock process contributes much more to consumption and investment fluctuations than does the preference shock process.

15 When $\sigma=0$ (the expected utility, rational expectations case) we can scale the state variables to account for geometric growth without affecting the subsequent analysis. However, when $\sigma<0$, the same transformation has the effect of imposing a time-varying risk adjustment. This problem does not arise when the single period utility function has a different form, say logarithmic. In order to preserve the tractability of the quadratic specification, we have decided to proceed despite this problem. 
Table 2

Parameter Estimates

$\begin{array}{crr} & \text { Habit } & \text { No habit } \\ \text { risk free rate } & .025 & .025 \\ \beta & .997 & .997 \\ \delta_{h} & .629 & \\ \lambda & .994 & 0 \\ \alpha & .851 & .945 \\ \phi_{1} & 1.379 & 1.284 \\ \phi_{2} & -.386 & -.298 \\ \mu_{d} & 13.758 & 13.716 \\ c_{b} & .018 & .003 \\ c_{d} & .205 & .206 \\ 2 \times \operatorname{LogLikelihood} & 755.24 & 749.49\end{array}$

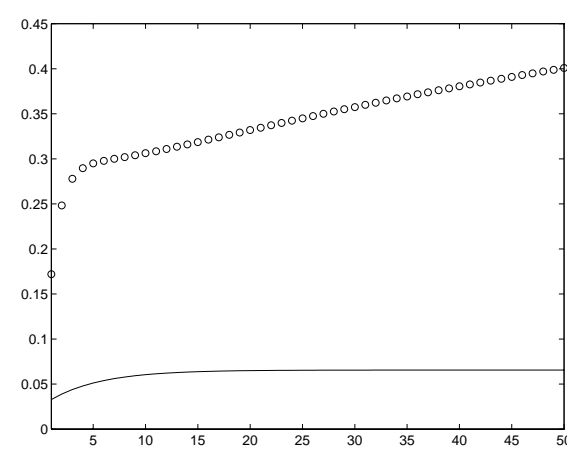

Figure 3a. Impulse response of investment (circles) and consumption (line) to innovation in endowment, at maximum likelihood estimate of habit persistence.

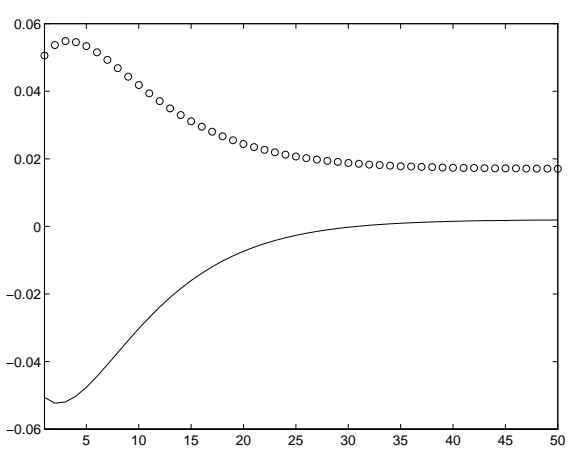

Figure 3b. Impulse response of investment (circles) and consumption (line) to innovation in preference shock, at maximum likelihood estimate of habit persistence. 


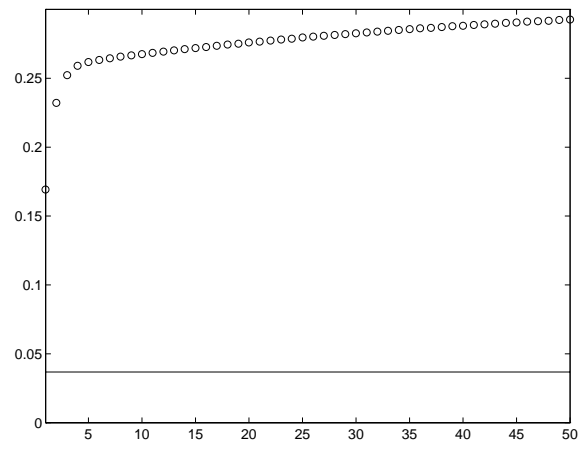

Figure 4a. Impulse response of investment (circles) and consumption (line) to innovation in endowment, no habit persistence.

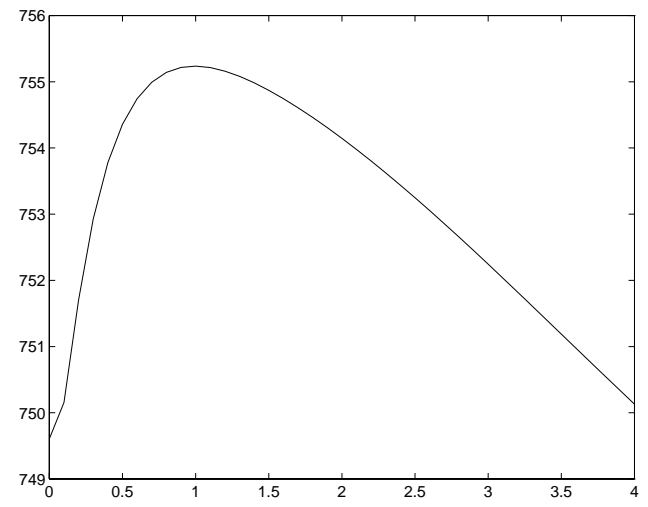

Figure 5a. Twice log likelihood, the ordinate, as a function of $\lambda$ (other parameters being concentrated out).

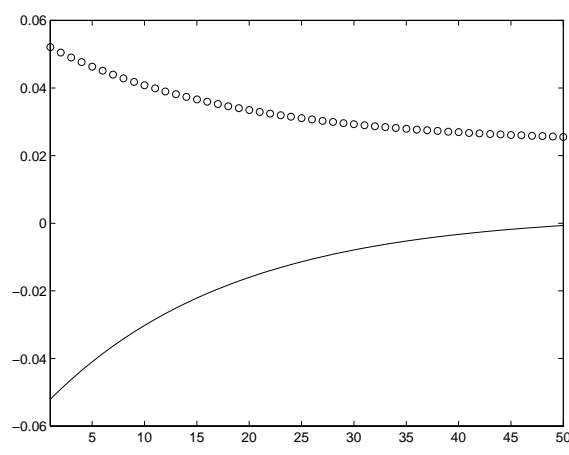

Figure 4b. Impulse response of investment (circles) and consumption (line) to innovation in preference shock, no habit persistence.

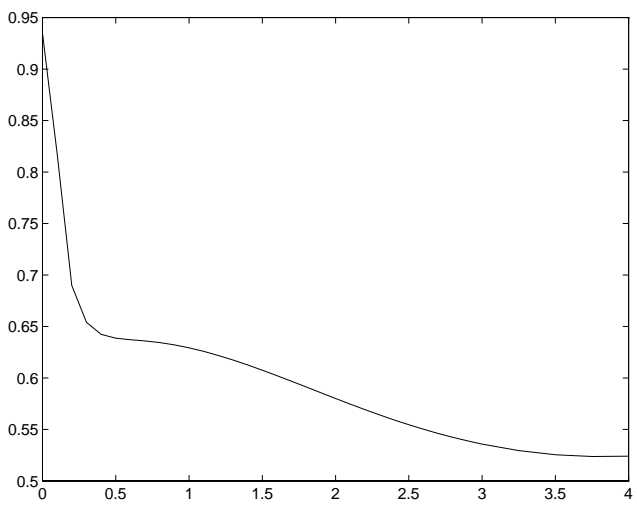

Figure 5b. Maximum likelihood $\delta_{h}$, the coordinate, as a function of $\lambda$, the ordinate.

To assess the statistical evidence for habit persistence, in Figure 5a we graph twice the concentrated $\log$ likelihood function as a function of the habit persistence parameter. Notice the asymmetry of this function, which has a much steeper descent towards zero. A likelihood-based confidence interval can be deduced by comparing the deterioration in the likelihood to critical values obtained from the chi-square one distribution. Thus, while values of $\lambda$ near zero are implausible, values considerably larger than the maximum are harder to dismiss. ${ }^{16}$ Figure $5 b$ shows the values of the depreciation parameter $\delta_{h}$ as a

16 The parameter $\delta_{h}$ is not identified when $\lambda=0$. 
function of the $\lambda$ obtained after concentrating the likelihood function. Estimates of the depreciation parameter increase as $\lambda$ approaches zero, but remain around .6, within the more plausible range of $\lambda$ 's.

We put our estimates of the habit persistence parameters, $\lambda$ and $\delta_{h}$, into perspective by comparing them with ones emerging from other empirical studies of aggregate U.S. data. Heaton (1993) finds a comparable value of $\lambda$, but a higher depreciation factor $\delta_{h}$ using a permanent income model without preference shocks fit to consumption. Heaton also notes that his $\delta_{h}$ is estimated very imprecisely. ${ }^{17}$ As an extension to this work, Heaton (1995) estimates a power utility, habit persistence model using consumption and asset market data. In this alternative formulation, he provides evidence for larger values of $\lambda$ and a larger depreciation factor $\delta_{h}$. Again the estimate of $\delta_{h}$ has a large standard error. From Heaton's work, we see that more pronounced habit persitence is estimated only when it is offset in the short run by local durability, a source of dynamics that we ignore. Recently, Boldrin, Christiano and Fisher (1995) find smaller values of $\lambda$ and $\delta_{h}$ than ours, although they model production in a different and maybe more interesting way than we. In contrast to Heaton (1995) and Boldrin, Christiano and Fisher (1995), our estimates of habit persistence embody no asset market implications beyond one for the risk free interest rate.

\section{Asset Pricing}

For the purposes of decentralization, we regard the robust (or risk-sensitive) solution to the permanent income model as the solution to an optimal resource allocation problem. This view point permits us to compute the equilibrium stochastic process of quantities before deducing the prices that clear a competitive security markets. We follow Lucas (1978) in assuming a large number of identical agents who trade in security markets. We can price assets by treating the consumption process that solves the robust permanent income model as though it were an endowment process. Because agents are identical, equilibrium prices become shadow prices that leave consumers content with that 'endowment process.' The single agent quality of the economy lets us compute (shadow) prices of a full array of securities without altering the aggregate consumption and investment processes. The pricing implications under robustness are slightly different than those under risk-sensitivity. We will proceed in this section by assuming risk-sensitivity and pointing out where the analysis would differ under robustness.

The state for the model is $x_{t}=\left[\begin{array}{lll}h_{t-1} & k_{t-1} & z_{t}^{\prime}\end{array}\right]^{\prime}$. The equilibrium consumption and service processes can be represented as $c_{t}^{e}=S_{c} x_{t}, s_{t}^{e}=S_{s} x_{t}$. Represent the endowment and preference shock processes as $d_{t}=S_{d} x_{t}, b_{t}=S_{b} x_{t}$. The equilibrium law of motion for

17 Like Christiano, Eichenbaum and Marshall (1991), Heaton (1993) also studies the implications of time aggregation, which we abstract from, and at the same time he allows for local durability in a continuoustime formulation of the model. 
the state has representation

$$
x_{t+1}=A^{o} x_{t}+C w_{t+1} .
$$

The value function at the optimal allocation can be represented as $U_{t}^{e}=x_{t}^{\prime} \Omega x_{t}+\rho$ where

$$
\begin{gathered}
\Omega=-\left(S_{s}-S_{b}\right)^{\prime}\left(S_{s}-S_{b}\right) / 2+\beta \hat{\Omega} \\
\rho=\beta \hat{\rho},
\end{gathered}
$$

and $\hat{\Omega}$ satisfies (5a), with $A$ evaluated at $A^{\circ}$.

\section{Key subgradient inequality}

We begin our analysis of asset pricing by computing the current time $t$ price of a statecontingent claim to utility $U_{t+1}$ tomorrow. This component of pricing is trivial when preferences are represented as the usual recursive version of the von-Morgenstern specification, but is nontrivial in the case of risk sensitivity. The pricing of state-contingent utility will be a key ingredient for pricing state-continent consumption services tomorrow and ultimately for the pricing of multi-period securities that are direct claims on consumption goods. Let $s_{t}$ be any service process measurable with respect to $J_{t}$, and $U_{t}$ be the associated utility index. For purposes of valuation, Appendix A establishes the following subgradient inequality:

$$
\mathcal{R}_{t}\left(U_{t+1}\right)-\mathcal{R}_{t}\left(U_{t+1}^{e}\right) \leq \mathcal{T}_{t} U_{t+1}-\mathcal{T}_{t} U_{t+1}^{e}
$$

where

$$
\mathcal{T}_{t} U_{t+1} \equiv E\left(V_{t+1} U_{t+1} \mid J_{t}\right) / E\left(V_{t+1} \mid J_{t}\right)
$$

and

$$
V_{t+1} \equiv \exp \left(\sigma U_{t+1}^{e} / 2\right)
$$

As elaborated further below, the operator $\mathcal{T}_{t}$ acts much like a conditional expectation. ${ }^{18}$ Combining (31) with the familiar gradient inequality for quadratic functions, it follows that

$$
U_{t}-U_{t}^{e} \leq\left(s_{t}-s_{t}^{e}\right) \mathcal{M}_{t}^{s}+\beta \mathcal{T}_{t}\left(U_{t+1}-U_{t+1}^{e}\right)
$$

where

18 Depicting prices of derivative claims using distorted expectations is a common technique in asset pricing (e.g., see Harrison and Kreps (1979)). In our investigation and in Epstein and Wang (1994), the distortion is also needed to price state-contingent utility. 


$$
\mathcal{M}_{t}^{s} \equiv\left(b_{t}-s_{t}^{e}\right)
$$

If we regard the marginal utility of services $\mathcal{M}_{t}^{s}$ as the price for time $t$ services, then (34) states that any pair $\left(s_{t}, U_{t+1}\right)$ that is preferred to $\left(s_{t}^{e}, U_{t+1}^{e}\right)$ costs more at time $t$. This justifies treating $\mathcal{M}_{t}^{s}$ as the equilibrium time $t$ price of services, and using $\beta \mathcal{T}_{t}$ to value time $t+1$ state-contingent utility.

The $\mathcal{T}_{t}$ operator can be computed as the conditional expectation of the state in the transformed transition equation:

$$
x_{t+1}=\hat{A} x_{t}+\hat{C} w_{t+1}
$$

where $\hat{C}$ satisfies

$$
\hat{C} \hat{C}^{\prime}=C\left(I-\sigma C^{\prime} \Omega C\right)^{-1} C^{\prime},
$$

and $\hat{A}$ is given by (12). Given the matrices $\hat{A}$ and $\hat{C}$, asset prices can be computed using the algorithms described in Hansen and Sargent (1996). Formula (37) shows that when $\sigma<0$ and $\Omega$ is negative semidefinite, the conditional variance associated with the operator $\mathcal{T}_{t}$ is always greater than or equal to $C C^{\prime}$, because an identity matrix is replaced by a larger matrix $\left(I-\sigma C^{\prime} \Omega C\right)^{-1}$. Thus, to interpret $\mathcal{T}_{t}$ as a conditional expectation operator requires both a pessimistic assignment of the conditional mean for the future state vector and an increase in its conditional variance. ${ }^{19}$

We can interchange the risk sensitivity and the uncertainty aversion interpretations of the optimal resource allocation problem. As shown by Epstein and Wang (1994), equilibrium asset prices can be deduced by referring to the 'pessimistic beliefs' that implement optimal decisions. For the uncertainty aversion interpretation, the counterpart to the $\mathcal{T}_{t}$ operator is the distorted conditional expectation operator, call it $\tilde{E}_{t}$, induced by the state transition equation of formula (11). This transition law distorts the conditional mean, but not the conditional variance. ${ }^{20}$

\section{Pricing multi-period streams}

The valuation of the state-contingent utility can be used to evaluate future consumption services. Construct a family of operators by sequential application of $\mathcal{T}_{t}$ :

$$
\mathcal{S}_{t, \tau}=\mathcal{T}_{t} \mathcal{T}_{t+1} \ldots \mathcal{T}_{t+\tau-1}
$$

\footnotetext{
19 It follows from James (1992) that this covariance correction vanishes in the continuous time formulation of the problem. Instead the original covariance structure is used.

20 Epstein and Wang (1994) consider different ways of introducing Knightian uncertainty, including ones in which there is an important difference between the game with time zero commitment and the game with sequential choice. Their specification of Knightian uncertainty can result in two-person games in which the 'beliefs' are not unique. This leads them to a form of price indeterminacy, which they link to empirical findings of excess volatility. In our setup, the 'beliefs' turn out to be unique and price indeterminacy is absent.
} 
where $\mathcal{S}_{t, 0}$ is the identity map. Like $\mathcal{T}_{t}, \mathcal{S}_{t, \tau}$ can be interpreted as a conditional expectation under a transformed conditional probability measure except that $\mathcal{S}_{t, \tau}$ is a time $t$ conditional expectation applied to random variables that are measurable with respect to $J_{t+\tau}$.

In the permanent income model below, the consumption good is a bundle of claims to future consumption services. We can use the equilibrium prices of services to deduce corresponding prices of consumption goods. Thus, consider any process $\left\{s_{t}\right\}$ in $L_{0}$, and let $\left\{U_{t}\right\}$ denote the associated utility process. Let $\left\{U_{t}^{e}\right\}$ denote the utility process associated with the equilibrium service process $\left\{s_{t}^{e}\right\}$. Then by iterating on (34), we find

$$
U_{t}-U_{t}^{e} \leq \sum_{\tau=0}^{\infty} \beta^{\tau} \mathcal{S}_{t, \tau}\left(\mathcal{M}_{t+\tau}^{s} s_{t+\tau}\right)-\sum_{\tau=0}^{\infty} \beta^{\tau} \mathcal{S}_{t, \tau}\left(\mathcal{M}_{t+\tau}^{s} s_{t+\tau}^{e}\right)
$$

Inequality (39) says that whenever $\left\{s_{t}\right\}$ is strictly preferred to $\left\{s_{t}^{e}\right\}$ as reflected by the associated time zero utility index, $\left(U_{t}>U_{t}^{e}\right)$, it also costs more. Hence $\left\{s_{t}^{e}\right\}$ is a solution to the consumer's intertemporal optimization problem when the time $t$ value of $\left\{s_{t}\right\}$ is computed according to the formula $\sum_{\tau=0}^{\infty} \beta^{\tau} \mathcal{S}_{t, \tau}\left(\mathcal{M}_{t+\tau}^{s} s_{t+\tau}\right)$. This justifies regarding this sum as the price of an asset offering a claim to the stream of services $\left\{s_{t}\right\}$.

If services are not traded 'unbundled', but only as bundles of state and date contingent claims, via the consumption goods, then what we really want is a consumption goods counterpart to $(39)$, namely:

$$
U_{t}-U_{t}^{e} \leq \sum_{\tau=0}^{\infty} \beta^{\tau} \mathcal{S}_{t, \tau}\left(\mathcal{M}_{t+\tau}^{c} c_{t+\tau}\right)-\sum_{\tau=0}^{\infty} \beta^{\tau} \mathcal{S}_{t, \tau}\left(\mathcal{M}_{t+\tau}^{c} c_{t+\tau}^{e}\right)
$$

A formula for the indirect marginal utility of consumption is deduced by ascertaining the implicit service flow associated with that a unit of consumption and then pricing that intertemporal bundle. Using this argument, it follows that $\mathcal{M}_{t}^{c}=M_{c} x_{t}$ where:

$$
M_{c} \equiv\left[(1+\lambda)+\left(1-\delta_{h}\right) \sum_{\tau=1}^{\infty} \beta^{\tau}\left(\delta_{h}\right)^{\tau}(-\lambda)(\hat{A})^{\tau}\right]\left(S_{b}-S_{s}\right)
$$

\section{Single-period security pricing}

A large body of empirical research has focused on pricing one-period securities. Imagine purchasing a security at time $t$ at a price $q_{t}$, holding it for one time period, then collecting the dividend and selling it at time $t+1$ for a total payoff $p_{t+1}$ of the consumption good. The payoff and price should satisfy:

$$
q_{t}=\mathcal{T}_{t}\left\{\left[\beta \mathcal{M}_{t+1}^{c} / \mathcal{M}_{t}^{c}\right] p_{t+1},\right\}
$$


where $\mathcal{M}_{t}^{c}=M_{c} x_{t}$ is the marginal utility of consumption and the formula for $M_{c}$ is given in (41). Under robustness, the price-payoff relationship would be given by:

$$
q_{t}=\tilde{E}_{t}\left\{\left[\beta \mathcal{M}_{t+1}^{c} / \mathcal{M}_{t}^{c}\right] p_{t+1},\right\}
$$

where $\tilde{E}_{t}$ is the distorted conditional expectations operator described above. A formula for $q_{t}$ in terms of the original conditional expectation operator is:

$$
q_{t}=E\left(m_{t+1, t} p_{t+1} \mid J_{t}\right)
$$

where the exact specification of $m_{t+1, t}$ will depend whether the robustness or the risksensitivity is adopted. The two alternatives will be explored in the next section. The random variable $m_{t+1, t}$ has an interpretation as a one-period stochastic discount factor, or alternatively as an equilibrium intertemporal marginal rate of substitution for the consumption good. The next section will show how risk-sensitivity and uncertainty aversion are reflected in the usual measure of the intertemporal marginal rate of substitution being scaled by a random variable (that depends on the interpretation - robustness or risksensitivity) with conditional expectation one. We use this multiplicative adjustment to the stochastic discount factor to increase its variability and to enhance risk premia.

From the one-period stochastic discount factor, we can easily deduce the 'market price of risk.' For simplicity, think of a one period payoff on an asset as a bundle of two attributes: its conditional mean and its conditional standard deviation. In our environment, these two attributes only partially describe asset payoffs. Furthermore, we cannot extract unique prices of the attributes, in part because one of the attributes, the standard deviation, is a nonlinear function of the asset payoff. Nevertheless, like any stochastic discount factor model, ours conveys information about how these attributes are valued (see Hansen and Jagannathan, 1991). To see this, consider the covariance decomposition of the right-hand side of (42):

$$
q_{t}=E_{t}\left(p_{t+1}\right) E_{t}\left(m_{t+1}\right)+\operatorname{cov}_{t}\left(m_{t+1}, p_{t+1}\right),
$$

where $\operatorname{cov}_{t}$ denotes the covariance conditioned on time $t$ information. Applying the Cauchy-Schwarz Inequality, we obtain the price bound:

$$
q_{t} \geq E_{t}\left(p_{t+1}\right) E_{t}\left(m_{t+1}\right)-s t d_{t}\left(m_{t+1}\right) s t d_{t}\left(p_{t+1}\right) .
$$

where $s t d_{t}$ denotes the standard deviation conditioned at time $t$. Along the so called 'efficient frontier,' the 'price of risk' relative to expected return is given by the ratio: $s t d_{t}\left(m_{t+1, t}\right) / E_{t}\left(m_{t+1, t}\right)$ which is commonly referred to as the market price of risk. This ratio is one way to encode information about how risk averse consumers are at the equilibrium consumption process. ${ }^{21}$ Appendix $\mathrm{C}$ describes how to compute the stochastic process for the market price of risk when $\sigma$ is negative under risk-sensitivity.

21 Gallant, Hansen, and Tauchen (1990), Hansen and Jagannathan (1991) and Cochrane and Hansen 


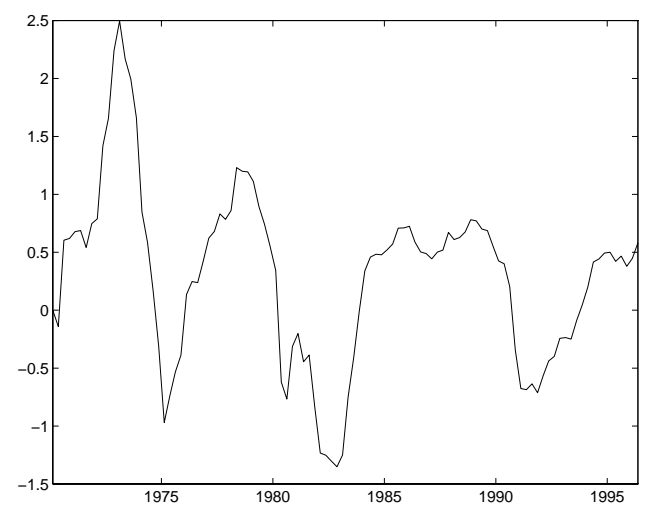

Figure 6a. Estimated process for endowment, $d_{t}-\mu_{d}$.

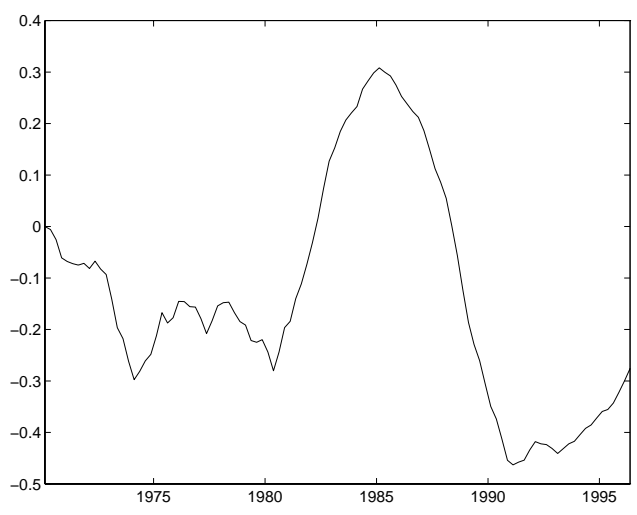

Figure 6b. Estimated process for the preference shock process, $b_{t}-\mu_{b}$.

\section{Quantifying Robustness from the Market Price of Risk}

Because it is not identified from data on consumption and investment, other information must be used to restrict the risk sensitivity parameter. In this section, we study how risk sensitivity alters the predicted market price of risk. We then exploit the connection between risk sensitivity and Knightian uncertainty by computing the magnitude of the specification errors needed to generate implications comparable to various settings of the parameter $\sigma$. In particular, we show how allowing for mistakes transmits to the equilibrium market price of risk. We are attracted to the interpretation in terms of robustness as a way of confronting an observation of Weil (1989), who noted how market prices of risk can be enhanced by risk sensitivity, but at the cost of making the implied risk aversion 'extreme.' Risk aversion has typically been measured by studying choice problems with unique specifications of the probability laws. That our risk sensitivity parameter has a nearly equivalent interpretation as reflecting aversion to uncertainty raises hopes for reinterpreting implausibly large estimates of risk aversion as coming partly from a 'preference for robustness.'

Market price of risk

While the risk-sensitivity parameter $\sigma$ and the preference curvature parameter $\mu_{b}$ are

(1992) interpret the equity premium puzzle as the large market price of risk implied by asset market date. The market price of risk can be expressed as the least upper bound on Sharpe ratios $\frac{\left|E_{t} r_{t+1}-r_{t}^{f}\right|}{s t d_{t}\left(r_{t}+1\right)}$ where $r_{t+1}$ is a one-period return and $r_{t}^{f}$ is the one-period riskless return. Thus the Sharpe ratio for the one-period return on equity gives a lower bound on the market price of risk. 
not identifiable from quantity data, we now show that they affect the market price of risk. In Tables $3 \mathrm{a}$ and $3 \mathrm{~b}$, we report median market prices of risk as functions of the risk sensitivity parameter for three choices of $\mu_{b}$. The tables are constructed using the implied state vectors obtained by applying the Kalman filter. Where $y_{t}=\left[\begin{array}{ll}c_{t} & i_{t}\end{array}\right]^{\prime}$, and $x_{t}=\left[\begin{array}{llllll}h_{t-1} & k_{t-1} & d_{t}^{*} & d_{t-1}^{*} & b_{t}^{*} & b_{t-1}^{*}\end{array}\right]^{\prime}$, we used the Kalman filter to compute $E\left(x_{t} \mid y_{t}, y_{t-1}, \ldots, y_{1}\right)$ for each time $t$ in our sample. It can be shown that the conditional covariance of the time $t$ state vector given time $t$ information converges to zero, implying that the 'hidden' states should be approximately revealed by the observations. Deviations around the means of the implied preference and endowment shift processes under habit persistence are graphed in Figure 6. We used these fitted states to calculate the median market price of risk over the sample. In Tables $3 \mathrm{a}$ and $3 \mathrm{~b}$, we report results for the model estimated with and without habit persistence, respectively. The tables show how we can achieve a 'target' market price of risk with alternative $\left(\sigma, \mu_{b}\right)$ pairs.

Table 3a

Median Market Price of Risk (with habit persistence)

\begin{tabular}{|c|c|c|c|c|c|}
\hline$\mu_{b}$ & $\sigma:$ & 0 & -.0001 & -.0002 & -.0003 \\
\hline 18 & & 0.0403 & 0.05 & 0.0715 & 0.0873 \\
\hline 24 & & 0.0145 & 0.0578 & 0.1014 & 0.1454 \\
\hline 30 & & 0.0088 & 0.0800 & 0.1519 & 0.2251 \\
\hline 36 & & 0.0064 & 0.1054 & 0.2062 & 0.3102 \\
\hline
\end{tabular}

Table $3 b$

Median Market Price of Risk (no habit persistence, $\lambda=0$ )

\begin{tabular}{|c|c|c|c|c|c|}
\hline$u$ & $\sigma:$ & 0 & -.0001 & -.0002 & -.0003 \\
\hline 10 & & 0.0208 & 0.0280 & 0.0352 & 0.0425 \\
\hline 24 & & 0.0072 & 0.0280 & 0.0487 & 0.0696 \\
\hline & & 0.0044 & 0.0387 & 0.0730 & 0.1076 \\
\hline 36 & & 0.0031 & 0.0510 & 0.0990 & 0.1474 \\
\hline
\end{tabular}

Given our arguably high value of the risk free rate (2.5\% per annum) and sampling error in estimates of the market price of risk, model predictions in the range of $.075-.150$ seem a reasonable "target." 22 Thus in the absence of risk sensitivity, for the $\mu_{b}$ specifications we consider, the market prices of risk are very small. The market price of risk can be

22 It is known from the work of Hansen and Jagannathan (1991) that achieving a market price of risk target is weaker than satisfying the consumption Euler equation. For example, we have not enabled the 
raised by reducing further the parameter $\mu_{b}$, but at the cost of enhancing the probability of satiation in the quadratic preference ordering. But increasing $|\sigma|$ pushes the model predictions towards more empirically plausible market prices of risk without altering the satiation probabilities. ${ }^{23}$ Roughly speaking, introducing habit persistence doubles the market price of risk across all of the $\left(\mu_{b}, \sigma\right)$ specifications we study. This conclusion from Table $3 \mathrm{~b}$ emerges from the estimates from the second (No Habit Persistence) column of Table 2. There the parameters governing the exogenous dynamics are adjusted to match the temporal covariations of consumption and investment as closely as possible.

Holding fixed $\sigma$ and increasing the preference translation parameter $\mu_{b}$ also enhances the market price of risk except when $\sigma$ is close to zero. To understand this finding, note that the stochastic discount factor can be represented as the product

$$
m_{t+1, t}=m_{t+1, t}^{f} m_{t+1, t}^{r}
$$

where

$$
m_{t+1, t}^{f} \equiv \beta \frac{\mathcal{M}_{t+1}^{c}}{\mathcal{M}_{t}^{c}}
$$

is the 'familiar' intertemporal marginal rate of substitution in the absence of risk sensitivity and

$$
m_{t+1, t}^{r} \equiv \frac{\exp \left(\sigma U_{t+1}^{e} / 2\right)}{E\left[\exp \left(\sigma U_{t+1}^{e} / 2\right) \mid J_{t}\right]} .
$$

(See Appendix $\mathrm{C}$ for an explicit formula for $m_{t+1, t}$ in terms of the equilibrium laws of motion.) When $\sigma=0$ this second term is one, and it always has conditional expectation equal to one. The latter property is what permits us to interpret this second factor as a pessimistic 'distortion' of the conditional expectation operator. Finally, recall that the market price of risk is simply the (conditional) standard deviation of $m_{t+1, t}$ divided by its (conditional) mean.

When $\mu_{b}$ is increased and $\sigma=0$, the single-period utility function is closer to being linear (risk neutral) over the empirically relevant portion of its domain. As a consequence, the market price of risk decreases as $\mu_{b}$ is increased (see the first columns of Tables 3a and $3 \mathrm{~b})$.

model to explain one of the glaring empirical failures of consumption-based asset pricing models: the observed lack of correlation between the implied intertemporal marginal rates of substitution and stock market returns. For a description of how to build statistical tests based on market price of risk targets, see Burnside (1994), Cecchetti, Lamb and Mark (1994), and Hansen, Heaton and Luttmer (1995).

23 It can be argued that risk sensitivity is simply repairing a defect in quadratic preferences, a criticism to which we are certainly vulnerable in this paper. The usual measure of relative risk aversion in the absence of habit persistence is $\frac{-U^{\prime \prime}(c)}{U^{\prime}(c) c}$. In the case of our quadratic preferences, this is given by $\frac{1}{(b-c) c}$, which requires that the bliss point process be very close to the consumption process to attain a risk aversion coefficient even as big as unity. For an investigation of risk sensitive preferences and logarithmic utility, see Tallarini (1996). 
Consider next cases in which $\left\{m_{t+1, t}^{f}\right\}$ is much smoother than $\left\{m_{t+1, t}^{r}\right\}$, so that the market price of risk is approximately $s t d\left(m_{t+1, t}^{r} \mid J_{t}\right)$. The (conditional) standard deviation of $\left\{m_{t+1, t}^{r}\right\}$ will be large when the distortion in the conditional expectation operator is large. As $\mu_{b}$ increases, the representative consumer's consumption is moved further away from his ideal point and hence the scope for pessimism is more pronounced. Thus increasing $\mu_{b}$ enhances the market price of risk.

More generally, the overall impact of increasing $\mu_{b}$ for a fixed $\sigma$ is ambiguous except when $\sigma=0$ and depends on the particular features of the calibrated economy. For the calculations reported in Tables $3 \mathrm{a}$ and $3 \mathrm{~b}$, the median market price of risk always increases with $\mu_{b}$ except when $\mu_{b}=0$.

\section{Market price of risk and robustness}

As we have just seen, risk sensitivity introduces an additional (multiplicative) factor $m_{t+1, t}^{r}$ into the stochastic discount factor. This factor changes only slightly when risk sensitivity is reinterpreted as a preference for robustness. (See the remarks in the paragraphs following (37).) When interpreted as a preference for robustness, we can abstract from the covariance enhancement of the shocks. However, relative to those reported in Tables $3 \mathrm{a}$ and $3 \mathrm{~b}$, the numbers for the market price of risk barely change when computed assuming Knightian uncertainty rather than risk-sensitive preferences.

Let $m_{t+1, t}^{u}$ denote the resulting multiplicative factor, so that the composite stochastic discount factor is:

$$
m_{t+1, t}=m_{t+1, t}^{u} m_{t+1, t}^{f}
$$

To aid our understanding, suppose initially that $m_{t+1, t}^{f}$ is constant, so the market price of risk is given by:

$$
\operatorname{mpr}_{t}=\operatorname{std}\left(m_{t+1, t}^{u} \mid J_{t}\right) \text {. }
$$

The first columns of Tables $3 \mathrm{~A}$ and $3 \mathrm{~B}$ suggest that $m_{t+1, t}^{u}$ is indeed close to zero for the preference specification used in our calculations.

Under our particular specification of uncertainty aversion, recall that asset prices are computed using the 'pessimistic' view of tomorrow's shock vector: $w_{t+1}$ is normally distributed with conditional mean $\hat{v}_{t}$ and covariance matrix $I$ where $\hat{v}_{t}$ is computed from the solution to the two-person game. It follows that

$$
m_{t+1, t}^{u}=\frac{\exp \left[-\left(w_{t+1}-\hat{v}_{t}\right)^{\prime}\left(w_{t+1}-\hat{v}_{t}\right) / 2\right]}{\exp \left(-w_{t+1} w_{t+1} / 2\right)}
$$

which is the density ratio of the 'distorted' relative to the 'true' probability distribution. By a straightforward calculation, it follows that

$$
E_{t}\left[\left(m_{t+1, t}^{u}\right)^{2}\right]=\exp \left(\hat{v}_{t}^{\prime} \hat{v}_{t}\right)
$$


and by construction

$$
E_{t}\left(m_{t+1, t}^{u}\right)=1
$$

Therefore,

$$
\operatorname{std}\left(m_{t+1, t}^{u} \mid J_{t}\right)=\left[\exp \left(\hat{v}_{t}^{\prime} \hat{v}_{t}\right)-1\right]^{1 / 2} \approx\left|\hat{v}_{t}\right|
$$

for small distortions. In other words, the market price of risk is approximately equal to the magnitude of the time $t$ specification error. Our market price of risk calculations under uncertainty aversion are only slightly smaller than those computed under risk sensitivity due to the small variance adjustment associated with the operator $\mathcal{T}_{t}$.

Since the shocks are normalized to have unit variances, a conditional mean distortion of, say, $10 \%$ of the shock standard deviation results in a market price of risk of approximately .10 , assuming that there is no variation in the usually constructed stochastic discount factor. The fact that a mistake in forecasting $w_{t+1}$ could lead to a direct enhancement of the market price of risk by the magnitude of the mistake is perhaps not surprising. What is conveyed here is that concern for robustness approximately directs the associated pessimism to returns that are conditionally mean-standard deviation efficient.

More generally, as a small noise approximation, $\left|\hat{v}_{t}\right|$ is an upper bound on the approximate enhancement to the market price of risk caused by the concern for robustness. Given

the 'pessimistic' construction of $v_{t}$, we expect the two components $m_{t+1, t}^{u}$ and $m_{t+1, t}^{f}$ of the stochastic discount factor to be positively correlated. This upper bound is more nearly attained when the two terms are highly positively correlated.

Table $4 a$

Mean Measure of Uncertainty Aversion, $\rho_{b} *$ (with habit persistence)

\begin{tabular}{rcrrrr}
$\mu_{b}$ & $\sigma:$ & 0 & -.0001 & -.0002 & -.0003 \\
\hline 18 & 0 & 0.0166 & 0.0325 & 0.0479 \\
& $(0,0)$ & $(.0146, .0196)$ & $(.0284, .0385)$ & $(.0416, .0571)$ \\
24 & 0 & 0.0392 & 0.0781 & 0.1169 \\
& $(0,0)$ & $(.0368, .0425)$ & $(.0734, .0849)$ & $(.1097, .1270)$ \\
30 & 0 & 0.0630 & 0.1260 & 0.1888 \\
& $(0,0)$ & $(.0606, .0665)$ & $(.1211, .1328)$ & $(.1816, .1991)$ \\
36 & 0 & 0.0871 & 0.1742 & 0.2613 \\
& $(0,0)$ & $(.0847, .0905)$ & $(.1694, .1811)$ & $(.2540, .2716)$
\end{tabular}

* Note: minimum and maximum values are in parenthesis below each mean $\rho$. 


\begin{tabular}{|c|c|c|c|c|c|c|}
\hline \multirow[t]{2}{*}{ Mean } & $\mathrm{Me}$ & \multicolumn{4}{|c|}{ Table $4 \mathrm{~b}$} & habit persistence) \\
\hline & $\mu_{b}$ & $\sigma:$ & 0 & -.0001 & -.0002 & -.0003 \\
\hline \multirow{2}{*}{\multicolumn{2}{|c|}{18}} & & 0 & 0.0092 & 0.0179 & 0.0264 \\
\hline & & & $(0,0)$ & $(.0081, .0108)$ & $(.0157, .0213)$ & $(.0230, .0315)$ \\
\hline \multirow{2}{*}{\multicolumn{2}{|c|}{24}} & & 0 & 0.0216 & 0.0431 & 0.0645 \\
\hline & & & $(0,0)$ & $(.0203, .0235)$ & $(.0405, .0468)$ & $(.0605, .0701)$ \\
\hline \multirow{2}{*}{\multicolumn{2}{|c|}{30}} & & 0 & 0.0348 & 0.0695 & 0.1042 \\
\hline & & & $(0,0)$ & $(.0335, .0367)$ & $(.0668, .0733)$ & $(.1002, .1099)$ \\
\hline \multirow{2}{*}{\multicolumn{2}{|c|}{36}} & & 0 & 0.0481 & 0.0961 & 0.1442 \\
\hline & & & $(0,0)$ & $(.0467, .0500)$ & $(.0935, .0999)$ & $(.1402, .1499)$ \\
\hline
\end{tabular}

\section{Measuring Knightian uncertainty}

Using formula (10), we can represent and compute the two components of $\hat{v}_{t}$, denoted $\hat{v}_{d, t}$ and $\hat{v}_{b, t}$, associated with the innovation to the endowment shock and preference shock, respectively. From (10), these 'worst case' specification errors are linear functions of the current Markov state. We take as our measure of Knightian uncertainty in the $\ell$ shock $(\ell=b, d)$ :

$$
\rho_{\ell}\left(x_{t}\right)=\sqrt{(1-\beta) E_{t} \sum_{j=0}^{\infty} \beta j\left(\hat{v}_{\ell, t+j}\right)^{2}}, \quad \ell=d, b .
$$

We report this measure for both shocks in Tables $4 \mathrm{a}$ and $4 \mathrm{~b}$. Included in these tables are the sample means as well as the minima and maxima observed over the sample. Like the market prices of risk, these measures are evaluated at the estimated values of the shock processes $\left(d_{t}, b_{t}\right)$ over the estimation period. Notice that the Knightian uncertainty measures for the preference shock innovation are almost double those of the endowment shock innovation.

The square of our measure of Knightian uncertainty is a long-run average of the squared single-period specification errors. This long-run averaging reduces the amount of time variation, as is evident in the relatively narrow range of the computed $\rho_{\ell}\left(x_{t}\right)$ 's. It also suggests that the $\rho_{\ell}\left(x_{t}\right)$ 's are approximately equal to the $\left|\hat{v}_{\ell, t}\right|$ 's, which turns out to be a good approximation in practice. Recall from our previous discussion that the enhancement of the market price of risk caused by Knightian uncertainty is approximately $\left|\hat{v}_{t}\right|$. These numbers are apparently dominated by specification errors in the preference shock processes. 


\section{Intertemporal Mean-Risk tradeoffs}

The market price of risk reported above conveys information about the one-period tradeoff between the mean and standard deviation of asset returns as encoded in shadow prices. We now investigate the implied intertemporal tradeoff between means and standard deviations associated with our alternative configurations of $\mu_{b}$ and $\sigma$. Specifically, given a proportionate reduction in the endowment shock, we aim to compute what proportionate increase in the conditional mean of the endowment is required to keep the social planner on the same indifference curve. Initially we answer this question 'locally' by considering small interventions. This partly imitates local measures of risk aversion. However, local measures of risk aversion are often computed around certainty lines. In our case, we localize around the solution to the permanent income optimal resource allocation problem. Our localization permits us to depict risk-aversion as the ratio of two appropriately chosen intertemporal prices. Thus, like the market price of risk, our intertemporal measure of risk aversion also can be interpreted as a price ratio. We supplement this local experiment with a global one in which the standard deviation of the shock is set to zero. The intertemporal vantage point adopted in this section affects the character of the implied measures of risk aversion. The calculations will be conducted using the 'risk-sensitive' decentralization. A corresponding 'robust' decentralization gives rise to essentially the same numbers.

\section{Local measure of risk aversion}

We form a local intertemporal tradeoff between the standard deviation and the mean of the endowment about the equilibrium process for consumption and investment. Specifically, given a proportional enhancement of the endowment innovation in all future time periods, we aim to compute what proportional increase in the endowment is required to keep the social planner on the same indifference curve, at least locally. To perform this computation we attain two 'value expansions,' both of which we describe below. The firstorder terms or 'derivatives' in these expansions can be interpreted as prices of appropriately chosen infinitely lived securities.

We implement a 'local' modification in the state evolution equation by adopting the parameterization of the law of motion starting for $j \geq 0$ as

$$
x_{t+j+1}^{\epsilon}=A_{0} x_{t+j}^{\epsilon}+(C+\epsilon G) w_{t+1+j},
$$

where $\epsilon$ is a small positive scalar. A positive $\epsilon$ initiates a change in the innovation standard deviation starting with date $t+1$. Here the matrix $G$ is designed to select the endowment innovation. For example, it can be identical to $C$ except with zeroes in the preference shock entries. Let $U_{t}=W^{\epsilon}\left(x_{t}\right)$ denote the value function for resulting control problem indexed by $\epsilon$; we take $W^{0}$ as the value function for a baseline control problem (say the risk sensitive permanent income model). Let

$$
x_{t+1}=A_{0} x_{t}+C w_{t+1}
$$


be the corresponding $\epsilon=0$ state evolution equation when the optimal control law is imposed. We aim to compute an expansion of the form:

$$
W^{\epsilon}(x)=W^{0}(x)+\epsilon W_{d}(x)+o(\epsilon)
$$

where $o(\epsilon)$ converges to zero as $\epsilon$ tends to zero uniformly on compact subsets of the state space. We will derive an asset pricing formulation of $W_{d}$ that, among other uses, facilitates calculations.

A corresponding experiment delivers a 'robust control' expansion. Alter the intervention that takes place at time $t$ by introducing 'mistakes' in the conditional mean. Now suppose instead that starting for $j \geq 0$ we have:

$$
x_{t+j+1}^{\epsilon}=A_{0} x_{t+j}+(C+\epsilon G)\left(w_{t+1+j}+v_{t+j}\right),
$$

As before, the parameter $\sigma$ is used to restrain mistakes, rather than to make a risk adjustment in the utility recursion. This perturbed system gives rise an expansion that, from a quantitative vantage point, is virtually identical to that we report. The subsequent asset pricing interpretation also applies, provided that we use the prices for the 'robust' decentralization in place of the prices of the 'risk sensitive' decentralization.

Of course, $W^{\epsilon}$ is a translated quadratic function of the state vector. We write this function as:

$$
W^{\epsilon}(x)=x^{\prime} \Omega^{\epsilon} x+\rho^{\epsilon} .
$$

The function $W_{d}$ is quadratic:

$$
W_{d}(x)=x^{\prime} \Omega_{d} x+\rho_{d} .
$$

In effect, $\Omega_{d}$ is the derivative with respect to $\epsilon$ of the matrix function $\Omega^{\epsilon}$, evaluated at $\epsilon=0$. Similarly, $\rho_{d}$ is the derivative with respect to $\epsilon$ of the scalar function $\rho^{\epsilon}$. Computations of these derivatives are simplified by the fact that we can abstract from the role of optimization of the control vector for small changes in $\epsilon$. This familiar property follows from the first-order conditions satisfied by the optimal control law, which imply that the contribution to the value function expansion is second order in $\epsilon$. Hence we can compute the derivatives as if we are holding fixed the control law and hence the state evolution matrix $A_{0}$. The matrix $\Omega_{d}$ can be computed easily as the solution of a Sylvester equation. 


\section{Measuring risk aversion by asset pricing}

Holding fixed the equilibrium law of motion for consumption, $c_{t}^{0}=S_{c} x_{t}$, we can use our asset pricing formula to evaluate how utility responds to changes in $\epsilon$. To compute the desired 'derivative' of $U_{t}$ with respect to $\epsilon$, we begin by forming a new state vector process:

$$
x_{t+j}^{\epsilon}-x_{t+j}^{0}=\epsilon y_{t+j}
$$

where $\left\{y_{t}\right\}$ evolves according to

$$
y_{t+j+1}=A_{0} y_{t+j}+G w_{t+1+j}
$$

with $y_{t}=0$. Notice the linear scaling in $\epsilon$. A consumption process associated with $\epsilon>0$ is:

$$
c_{t+j}^{\epsilon}=c_{t+j}^{0}+\epsilon S_{c} y_{t+j} .
$$

It follows from our subgradient inequality that

$$
\frac{W^{\epsilon}\left(x_{t}\right)-W^{0}\left(x_{t}\right)}{\epsilon} \leq \sum_{j=1}^{\infty} \beta^{j} \mathcal{S}_{t, j}\left(\mathcal{M}_{t+j}^{c} S_{c} y_{t+j}\right)
$$

It can be verified that as $\epsilon$ declines to zero, this becomes an equality. Therefore, we can evaluate the desired 'derivative' by using the following asset pricing formula:

$$
\sum_{j=1}^{\infty} \beta^{j} \mathcal{S}_{t, j}\left(\mathcal{M}_{t+j}^{c} S_{c} y_{t+j}\right)
$$

This is the time $t$ price, scaled in units of marginal utility, of an infinitely-lived security with dividend $\left\{S_{c} y_{t+j}\right\} \cdot{ }^{24}$

To compute the local mean-risk tradeoff, we also estimate the utility change associated with a small change in the conditional mean of the endowment. We capture this small change as follows:

$$
\begin{aligned}
& x_{t+1}^{\delta}=A_{0} x_{t}+D x_{t}+C w_{t+1} \\
& x_{t+j+1}^{\delta}=A_{0} x_{t+j}^{\delta}+C w_{t+1+j},
\end{aligned}
$$

for $j=1,2, \ldots$. This envisions the change in the conditional expectation as occurring at date $t+1$ continuing into the future and leads us to the time $t$ value-function expansion:

$$
\hat{W}^{\delta}(x)=W^{0}(x)+\delta \hat{W}_{d}(x)+o(\delta) .
$$

24 To perform the computation, first form the state transition equation for the composite state $\left(x_{t}^{0} \quad y_{t}^{\prime}\right)$. The transition equation has a block diagonal state matrix with diagonal blocks $A_{0}$. The counterpart to $C$ is constructed by stacking $C$ on top of $G$. Consumption will be formed by using a matrix ( $S_{c} \quad 0$ ) and the dividend will be formed by $\left(\begin{array}{ll}0 & S_{c}\end{array}\right)$. Prices can now be computed recursively using a doubling algorithm. 
Here $\hat{W}_{d}$ is a quadratic function of the state vector, which we represent as $x^{\prime} \hat{\Omega}_{d} x$.

Imitating our earlier derivation, we form:

$$
x_{t+j}^{\delta}-x_{t+j}=\delta A_{0}^{j-1} D x_{t}
$$

Notice the linear scaling in $\delta$. The new consumption process can be expressed as:

$$
c_{t+j}^{\delta}=c_{t+j}^{0}+\delta S_{c} A_{0}^{j-1} D x_{t} .
$$

From our subgradient inequality (39),

$$
\frac{W^{\delta}\left(x_{t}\right)-W^{0}\left(x_{t}\right)}{\delta} \leq \sum_{j=1}^{\infty} \mathcal{S}_{t, j}\left(\mathcal{M}_{t+j}^{c} S_{c} A_{0}^{j-1} D x_{t}\right) .
$$

Again we can show that this subgradient is actually a gradient by driving $\delta$ to zero. Therefore, our target derivative is given by:

$$
\sum_{j=1}^{\infty} \mathcal{S}_{t, j}\left(\mathcal{M}_{t+j}^{c} S_{c} A_{0}^{j-1} D x_{t}\right)
$$

which is the (time $t$ util) price of an infinitely-lived security with dividend $\left\{S_{c} A_{0}^{j-1} D x_{t}\right\}$. Thus, $\left\{\hat{\Omega}_{d}\right\}$ solves a Sylvester equation.

Using our two expansions, the compensation measure is:

$$
\delta_{t}=-\frac{x_{t}^{\prime} \Omega_{d} x_{t}+\rho_{d}}{x_{t}^{\prime} \hat{\Omega}_{d} x_{t}},
$$

\begin{tabular}{|c|c|c|c|c|}
\hline$\mu_{b}$ & 0 & -.0001 & -.0002 & -.0003 \\
\hline$\overline{18}$ & 0.0312 & 0.0480 & 0.0801 & 0.1527 \\
\hline 24 & 0.0112 & 0.0543 & 0.1303 & 0.2970 \\
\hline 30 & 0.0068 & 0.0769 & 0.1997 & 0.4682 \\
\hline 36 & 0.0049 & 0.1021 & 0.2720 & 0.6432 \\
\hline
\end{tabular}

which we index by $t$ to accommodate the change in vantage point as time passes.

Table 5

Local Mean-Risk Trade-off 


\section{Table 6 Global Mean-Risk Trade-off}

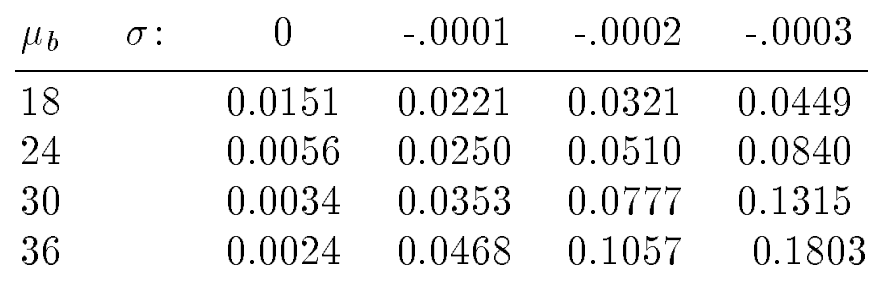

In Table 5, we report our (local) intertemporal measures of risk aversion. The effect of increasing (in absolute value) $\sigma$ has a stronger effect on the mean-risk trade-off than on the market price of risk (compare Table 5 to Table 3a). Increases in $\mu_{b}$ also have a slightly greater impact for the trade-off calculation. ${ }^{25}$

We next verify the local nature of these computations by considering the following experiment. Let $\epsilon=-1$, which sets to zero the shock variance for the endowment process. By extrapolating the local measures reported in Table 5, the entries in this table should convey what fraction of the endowment the consumer would be willing to forego to achieve this reduction in volatility. Such an inquiry relates to Lucas's quantification of the welfare costs to fluctuations, except that we are using a permanent income model that permits investment (see also Obstfeld, 1994 and Tallarini, 1996). From this vantage point, the numbers in Table 5 look to be enormous, particularly for the larger (in absolute value) specifications of $\sigma$. However, that extrapolation of our local measure turns out to be misleading. To see this, in Table 6 we report global numbers for the $\epsilon=-1$ experiment that hold fixed the permanent income decision rule for the two competing specifications of the endowment process. The global mean-risk tradeoffs are much smaller by a factor ranging from two to four. Nevertheless, the tradeoffs remain quite large, except when $\sigma$ is close to zero. ${ }^{26}$

25 Increasing the market price of risk by enlarging $\mu_{b}$ has the virtue of further reducing the probability of satiation. This would appear to increase the intertemporal substitutability of consumption. However, recall that $\mu_{b}$ does not appear in the permanent income decision rule. Thus, by design we have not changed the consumption-savings behavior of the consumer as we change $\mu_{b}$. On the other hand, some perverse implications 'off the equilibrium path' can occur for large values of $\mu_{b}$.

26 The global numbers would be enhanced a little if we reoptimize when setting the endowment shock to zero. The solution to linear-quadratic problem is unappealing in this context because with less uncertainty, capital ceases to be an attractive way to transform goods from one period to the next. In light of this, it seems crucial to reoptimize subject to a nonnegativity constraint on capital. Our imposition of the suboptimal 'permanent income' consumption rule diminishes the impact of this nonnegativity constraint while possibly misstating the global tradeoff. 


\section{Conclusions}

Robert E. Lucas, Jr. (1975) warned us about theorists bearing free parameters. Having heard Lucas, we devoted this paper to scrutinizing some of the implications for prices and quantities of a single additional parameter designed to quantify a preference for a particular type of robustness. By exploiting the connection between this type of robustness and the risk-sensitivity specification of Jacobson (1973) and Whittle (1990), we have shown how to decentralize dynamic, stochastic general equilibrium models with a risk sensitive representative consumer. Formulas for consumption, investment, and the risk-free interest rate are identical with ones coming from plain permanent income models. We presented formulas for the market price of risk, then applied them to account for the market price of risk observed in U.S. data.

Like Brock and LeBaron (1996), Brock and Hommes (1994), Cochrane (1989), Marcet and Sargent (1989), and Krusell and Smith (1996), we can regard the consumer-investors in our economy as making 'mistakes', but as managing them differently than do those in these authors' models. ${ }^{27}$ Our agents are very sophisticated in how they accommodate possible mistakes: they base decisions on worse-case scenarios, following Gilboa and Schmeidler (1989) and Epstein and Wang (1994).

In constrast to Cochrane (1989) and and Krusell and Smith (1996), for our permanent income economy, the quantity allocations are observationally equivalent to those in an economy in which no 'mistakes' are contemplated. This situation stems partly from the econometrician's ignorance of the subjective discount factor. Like Epstein and Wang (1994) and Melino and Epstein (1995), we focus on how aversion to mistakes transmits into security market prices. We find that a conditional mean 'mistake' of $x \%$ of the conditional standard deviation increases the market price of risk by approximately $x / 100$.

An ironic feature of our estimation procedure is that we ignore the possibility of our own misspecification errors while estimating a model whose agents' decisions are driven by their specification errors. A prime motivation for robust decision theory is to relax the literal interpretation of the control problem, and to regard the posited dynamic, stochastic constraints as approximations to a more complicated environment. It might be useful to put the agents and the econometrician on a more equal footing, by admitting specification errors into the econometric investigation.

We have concentrated on a robust interpretation of the permanent income model of

27 Cochrane's and Krusell and Smith's agents use decision rules that are perturbed by small amounts in arbitrary directions from optimal ones. Marcet and Sargent's agents correctly solve dynamic programming problems, but subject to subtly misspecified constraints: they use estimated transition laws (usually of the correct functional forms) which they mistakenly take as non-random and time-invariant. See Brock and LeBaron (1996), especially their footnote 2, for a lucid explanation of a class of models that mix 'adaptation' - to induce local instability near rational expectations equilibria - with enough 'rationality' to promote global attraction toward the vicinity of rational expectations. Brock and LeBaron and Brock and Hommes balance the tension between adaptation and rationality to mimic some interesting return and volume dynamics. 
consumption. The permanent income model seemed a natural starting point for exploring the consequences of robust decision theory, partly because of its simplicity. Recent work by Carroll (1992) has emphasized a departure from the permanent income model induced by precautionary savings, low discount factors, and big utility costs to zero consumption. ${ }^{28}$ As we have emphasized, our reinterpretation of the permanent income model also relies on smaller discount factors and precautionary savings. It does not, however, permit us to explore the ramifications of big utility costs to zero consumption, which is central to the work of Carroll (1992) and others. Recently, James (1992, 1995) and Campi and James (1995) demonstrate the connection between risk sensitivity and robustness using small noise expansions for control problems with nonquadratic criteria and nonlinear, stochastic evolution equations. These expansion methods provide a justification in terms of robustness for using risk sensitive control for a much richer class of decision problems. Recursive counterparts of these expansions will open the way to studying discounted, nonlinear robust control and its ramifications for consumption and savings.

We can be accused of taking the representative agent paradigm too seriously. We use the representative agent as a convenient starting point to understand the workings of risk sensitivity and robustness in decentralized economies. In other setups we know how heterogeneity of preferences and incomplete risk sharing affect investment behavior and the market price of risk. In our model (and Epstein and Wang's), agents agree on the amount and location of the Knightian uncertainty. Thus, models like ours can contribute an additional dimension upon which heterogeneity alters equilibrium quantities and prices.

\section{Appendix A: Subgradient Inequality}

This derives the subgradient inequality used for equilibrium pricing. Let $U^{e}$ denote the original nonpositive random utility index, $U$ any other nonpositive random utility index and $J$ a sigma algebra of events. We will show that

$$
\mathcal{R}(U)-\mathcal{R}\left(U^{e}\right) \leq E\left[V^{e}\left(U-U^{e}\right) \mid J\right] / E\left(V^{e} \mid J\right)
$$

where

$$
\begin{aligned}
\mathcal{R}(U) & \equiv(2 / \sigma) \log \{E[\exp (\sigma U / 2) \mid J] \\
V^{e} & \equiv \exp \left(\sigma U^{e} / 2\right) .
\end{aligned}
$$

We assume that $E\left[\exp \left(\sigma U^{e} / 2\right) \mid J\right]$ and hence $\mathcal{R}\left(U^{e}\right)$ is finite with probability one. Define $h \equiv U-U^{e}$, and let $\delta$ be any real number in $(0,1)$. Interpret $\delta$ as determining the

28 See Leland (1968) and Miller (1974) for important early contributions to the literature on precautionary saving. 
magnitude of a perturbation in direction $h$. In other words, the perturbation away from $U^{e}$ under consideration is $\delta h$.

By the convexity of the exponential function:

$$
\exp \left[\sigma\left(U^{e}+h \delta\right) / 2\right]-\exp \left(\sigma U^{e} / 2\right) \geq \delta h(\sigma / 2) V^{e} .
$$

This inequality remains true when computing expectations conditioned on $J$, although either side may be infinite:

$$
E\left\{\exp \left[\sigma\left(U^{e}+h \delta\right) / 2\right] \mid J\right\}-E\left\{\exp \left(\sigma U^{e} / 2\right) \mid J\right\} \geq \delta(\sigma / 2) E\left(V^{e} h \mid J\right) .
$$

Divide each side of $(50)$ by $E\left(V^{e} \mid J\right)$ :

$$
\begin{gathered}
E\left\{\exp \left[\sigma\left(U^{e}+h \delta\right) / 2\right] \mid J\right\} / E\left\{\exp \left(\sigma U^{e} / 2\right) \mid J\right\}-1 \geq \\
\delta(\sigma / 2) E\left(V^{e} h \mid J\right) / E\left(V^{e} \mid J\right) .
\end{gathered}
$$

Since $0<\delta<1,\left(U^{e}+h \delta\right)$ is a convex combination of $U^{e}$ and $U$ with weights $(1-\delta)$ and $\delta$ respectively. By the conditional version of the Hölder Inequality,

$$
\begin{aligned}
E\left\{\exp \left[\sigma\left(U^{e}+h \delta\right) / 2\right] \mid J\right\} & =E\left\{\left[\exp \left(\sigma U^{e} / 2\right)\right]^{1-\delta}[\exp (\sigma U / 2)]^{\delta} \mid J\right\} \\
& \leq\left\{E\left[\exp \left(\sigma U^{e} / 2\right) \mid J\right]\right\}^{1-\delta}\{E[\exp (\sigma U / 2) \mid J]\}^{\delta}
\end{aligned}
$$

Combining (51) and (52) and dividing by $\delta$, we have that

$$
\begin{aligned}
&(1 / \delta)\left\{E[\exp (\sigma U / 2) \mid J] / E\left(V^{e} \mid J\right)\right\}^{\delta}-1 \geq \\
&(\sigma / 2) E\left(V^{e} h \mid J\right) / E\left(V^{e} \mid J\right)
\end{aligned}
$$

To complete the derivation, we use the familiar approximation result for logarithms:

$$
\lim _{\delta \rightarrow 0}\left(\lambda^{\delta}-1\right) / \delta=\log (\lambda)
$$

where the limit is from above. (This limit can be verified by applying L'Hospital's Rule or by using the series expansion for $\exp [\delta \log (\lambda)])$. Taking limits of the left side of $(53)$ as $\delta$ declines to zero yields

$$
\log \{E[\exp (\sigma U / 2) \mid J]\}-\log \left[E\left(V^{e} \mid J\right)\right] \geq(\sigma / 2) E\left(V^{e} h \mid J\right) / E\left(V^{e} \mid J\right)
$$

The desired inequality (47) is obtained by multiplying both sides of (55) by the negative number $(2 / \sigma)$ and reversing the inequality. 


\section{Appendix B: Computing Prices for State-Contingent Utility}

In this appendix we provide a characterization of the operator $\mathcal{T}_{t}$ used in pricing statecontingent utility. The characterization relies on a restriction that the utility index $U_{t+1}^{e}$ be quadratic in a normally distributed state vector $x_{t+1}$. For notational convenience, we will suppress superscripts and subscripts.

Suppose that a utility index is quadratic in a normally distributed random vector $x \in$ $\mathbf{R}^{n}$ :

$$
U=x^{\prime} \Omega x+\rho
$$

where $\Omega$ is a negative semidefinite matrix and $\rho \leq 0$. In addition, suppose that

$$
x=\mu+C w
$$

where $w$ is normally distributed random vector with mean zero and covariance matrix $I$. Recall that $\mathcal{T}_{t}$ can be interpreted as a conditional expectation with a change of probability measure. In terms of the notation just developed, the new probability measure is constructed using $V / E V$ as a Radon-Nikodym derivative where

$$
V=\exp (\sigma U / 2) \propto \exp \left(\sigma w^{\prime} C^{\prime} \Omega C w / 2+\sigma w^{\prime} C^{\prime} \Omega \mu\right) .
$$

We can compute expectations with respect to the transformed measure as follows. Let $\phi$ be any bounded, Borel measurable function mapping $\mathbf{R}^{m} \rightarrow \mathbf{R}$. Then

$$
E[V \phi] / E V \propto \int \phi(w) \exp \left(\sigma w^{\prime} C^{\prime} \Omega C w / 2+\sigma w^{\prime} C^{\prime} \Omega \mu\right) \exp \left(-w^{\prime} w / 2\right) d w .
$$

Note that

$$
\begin{gathered}
\sigma w^{\prime} C^{\prime} \Omega C w / 2+\sigma w^{\prime} C^{\prime} \Omega \mu-w^{\prime} w / 2=-w^{\prime}\left(I-\sigma C^{\prime} \Omega C\right) w / 2+ \\
w^{\prime}\left(I-\sigma C^{\prime} \Omega C\right)\left(I-\sigma C^{\prime} \Omega C\right)^{-1} \sigma C^{\prime} \Omega \mu .
\end{gathered}
$$

Consequently, the operator on the left side of (59) can be evaluated by integrating $\phi$ with respect to a normal density with mean vector:

$$
\tilde{\mu} \equiv\left(I-\sigma C^{\prime} \Omega C\right)^{-1} \sigma C^{\prime} \Omega \mu
$$

and covariance matrix

$$
\tilde{\Sigma} \equiv\left(I-\sigma C^{\prime} \Omega C\right)^{-1} .
$$

The corresponding mean vector and covariance matrix for $x$ are $\mu+C \tilde{\mu}$ and $C \tilde{\Sigma} C^{\prime}$, respectively. The $\mathcal{T}_{t}$ operator will only be well defined so long as $\sigma C^{\prime} \Omega C<I$. 


\section{Appendix C}

\section{Computing the Conditional Variance of the Stochastic Discount Factor}

From Eq. (45), we know that $m_{t+1, t}$, the intertemporal marginal rate of substitution between time $t$ and time $t+1$ can be written as:

$$
m_{t+1, t}=\frac{\beta\left[\exp \left(\sigma U_{t+1}^{e} / 2\right) \nu^{\prime} \mathcal{M}_{t+1}^{c}\right]}{E\left\{\exp \left(\sigma U_{t+1}^{e} / 2\right) \mid J_{t}\right\} \nu^{\prime} \mathcal{M}_{t}^{c}}
$$

or as:

$$
m_{t+1, t}=\frac{\beta\left\{\exp \left[\sigma\left(x_{t+1}^{\prime} \Omega x_{t+1}+\rho\right) / 2\right] \nu^{\prime} M_{c} x_{t+1}\right\}}{\exp \left[\sigma\left(x_{t}^{\prime} \hat{\Omega} x_{t}+\hat{\rho}\right) / 2\right] \nu^{\prime} M_{c} x_{t}}
$$

where $\hat{\Omega}$ and $\hat{\rho}$ are given by (5). By applying the results of Appendix B we can compute the mean of $m_{t+1, t}$, conditional on information available at time $t$. The result can be written as

$$
E\left(m_{t+1,1} \mid J_{t}\right)=\beta\left(\nu^{\prime} M_{c} \hat{A} x_{t}\right) /\left(\nu^{\prime} M_{c} x_{t}\right)
$$

Our present goal is to compute the conditional second moment of $m_{t+1, t}$ as a means for computing its conditional variance. We will accomplish this by manipulating $m_{t+1, t}^{2}$ so that we can transform the probability measure as in Appendix B but with a different function $V$. We have

$$
m_{t+1, t}^{2}=\frac{\beta^{2}}{\left(\nu^{\prime} M_{c} x_{t}\right)^{2}} \frac{\exp \left[\sigma\left(x_{t+1}^{\prime} \Omega x_{t+1}+\rho\right)\right]}{\exp \left[\sigma\left(x_{t}^{\prime} \hat{\Omega} x_{t}+\hat{\rho}\right)\right]}\left(\nu^{\prime} M_{c} x_{t+1}\right)^{2} .
$$

ultiply the numerator and denominator by the time $t$ conditional mean of the exponential term in the numerator, $E\left\{\exp \left[\sigma\left(x_{t+1}^{\prime} \Omega x_{t+1}+\rho\right)\right] \mid J_{t}\right\}$. This gives us

$$
m_{t+1, t}^{2}=\frac{\left.\beta^{2} E\left\{\exp \left[\sigma\left(x_{t+1}^{\prime} \Omega x_{t+1}+\rho\right)\right] \mid J_{t}\right)\right\}}{\left(\nu^{\prime} M_{c} x_{t}\right)^{2} \exp \left[\sigma\left(x_{t}^{\prime} \hat{\Omega} x_{t}+\hat{\rho}\right)\right]} \frac{\exp \left[\sigma\left(x_{t+1}^{\prime} \Omega x_{t+1}+\rho\right)\right]\left(\nu^{\prime} M_{c} x_{t+1}\right)^{2}}{E\left\{\exp \left[\sigma\left(x_{t+1}^{\prime} \Omega x_{t+1}+\rho\right)\right] \mid J_{t}\right\}} .
$$

This conditional expectation can be computed by using a formula found in Jacobson (1973), only substituting $2 \sigma$ for $\sigma$ :

$$
\begin{aligned}
E\left\{\exp \left[\sigma\left(x_{t+1}^{\prime} \Omega x_{t+1}+\rho\right)\right] \mid J_{t}\right\} & =\left[\operatorname{det}\left(I-2 \sigma C^{\prime} \Omega C\right)\right]^{-1 / 2} \exp \left[\sigma\left(x_{t}^{\prime} \tilde{\Omega} x_{t}+\rho\right)\right] \\
& =\exp \left[\sigma\left(x_{t}^{\prime} \tilde{\Omega} x_{t}+\tilde{\rho}\right)\right]
\end{aligned}
$$

where $\tilde{\Omega} \equiv A^{\prime}\left[\Omega+2 \sigma \Omega C\left(I-2 \sigma C^{\prime} \Omega C\right)^{-1} C^{\prime} \Omega\right] A$ and $\tilde{\rho} \equiv-\frac{1}{2 \sigma} \log \operatorname{det}\left(I-2 \sigma C^{\prime} \Omega C\right)+\rho$. So we get that

$$
\frac{E\left\{\exp \left[\sigma\left(x_{t+1}^{\prime} \Omega x_{t+1}+\rho\right)\right] \mid J_{t}\right\}}{\exp \left[\sigma\left(x_{t}^{\prime} \hat{\Omega} x_{t}+\hat{\rho}\right)\right]}=\exp \left\{\sigma\left[x_{t}^{\prime}(\tilde{\Omega}-\hat{\Omega}) x_{t}+\tilde{\rho}-\hat{\rho}\right]\right\} .
$$


This gives us

$$
m_{t+1, t}^{2}=\frac{\beta^{2}}{\left(\nu^{\prime} M_{c} x_{t}\right)^{2}} \exp \left\{\sigma\left[x_{t}^{\prime}(\tilde{\Omega}-\hat{\Omega}) x_{t}+\tilde{\rho}-\hat{\rho}\right]\right\} \frac{\tilde{V}_{t+1}\left(\nu^{\prime} M_{c} x_{t+1}\right)^{2}}{E\left\{\tilde{V}_{t+1} \mid J_{t}\right\}}
$$

where $\tilde{V}_{t+1}=\exp \left[\left(\sigma\left(x_{t+1}^{\prime} \Omega x_{t+1}+\rho\right)\right]\right.$. So

$$
E\left(m_{t+1, t}^{2} \mid J_{t}\right)=\frac{\beta^{2}}{\left(\nu^{\prime} M_{c} x_{t}\right)^{2}}\left\{\exp \left\{\sigma\left[x_{t}^{\prime}(\tilde{\Omega}-\hat{\Omega}) x_{t}+\tilde{\rho}-\hat{\rho}\right]\right\} \tilde{\mathcal{T}}_{t}\left[\left(\nu^{\prime} M_{c} x_{t+1}\right)^{2}\right]\right.
$$

where $\tilde{\mathcal{T}}_{t}$ is the transformed conditional expectation operator for a $2 \sigma$ economy. We can evaluate the $\tilde{\mathcal{T}}_{t}$ term in the above expression using results from Appendix B:

$$
\tilde{\mathcal{T}}_{t}\left[\left(\nu^{\prime} M_{c} x_{t+1}\right)^{2}\right]=x_{t}^{\prime} \tilde{A}^{\prime} M_{c}^{\prime} \nu \nu^{\prime} M_{c} \tilde{A} x_{t}+\operatorname{trace}\left(\nu^{\prime} M_{c} \tilde{C} \tilde{C}^{\prime} M_{c}^{\prime} \nu\right)
$$

where

$$
\tilde{A} \equiv\left[I+2 \sigma C\left(I-2 \sigma C^{\prime} \Omega C\right)^{-1} C^{\prime} \Omega\right] A
$$

and

$$
\tilde{C} \tilde{C}^{\prime} \equiv C\left(I-2 \sigma C^{\prime} \Omega C\right)^{-1} C^{\prime}
$$

Finally, we know that the conditional variance of $m_{t+1, t}$ is given by its conditional second moment minus the square of its conditional mean. 


\section{References}

Becker, G.S. and K.M. Murphy (1988). 'A Theory of Rational Addiction'. Journal of Political Economy, 96, 675-700.

Bewley, T. (1977). 'The Permanent Income Hypothesis: A Theoretical Formulation'. Journal of Economic Theory, 16, 252-259.

Boldrin, M., L. J. Christiano, and J. D. M. Fisher (1995). 'Asset Pricing Lessons for Modeling Business Cycles'. Mimeo. NBER Working Paper No. 5262..

Brock, William A. and Cars Hommes (1994). 'Rational Routes to Randomness: Rationality in an Unstable Market with Information Costs Implies Chaos'. Mimeo. Department of Economics, University of Wisconsin at Madison.

Brock, William A. and Blake D. LeBaron (1996). 'A Dynamic Structural odel for Stock Return Volatility and Trading Volume'. Review of Economics and Statistics, Vol. LXXVIII, Vol. 1, February, pp. 94-110.

Burnside, C., (1994). 'Hansen-Jagannathan Bounds as Classical Tests of Asset Pricing Modesl'. Journal of Business and Economic Statistics, 12, 57-79.

Burnside, C., M. Eichenbaum, and S. Rebelo (1990). 'Labor Hoarding and the Real Business Cycle'. Mimeo. NBER Working Paper No. $3556 .$.

Campbell, John Y. (1987). 'Does Saving Anticipate Declining Labor Income? An Alternative Test of the Permanent Income Hypothesis'. Econometrica, 55:6, 1249-1273.

Campi, M. C. and M. R. James, (1995). 'Nonlinear Discrete-Time Risk-Sensitive Control'. International Journal of Robust Nonlinear Control, forthcoming.

Carroll, C.D. (1992). 'The Buffer-Stock Theory of Saving: Some Macroeconomic Evidence'. Brookings Papers on Economic Activity, 2, 61-156.

Cecchetti, S.G., P. Lam, and N.C. Mark, (1994). 'Testing Volatility Restrictions on Intertemporal Marginal Rates of Substitution Implied by Euler Equations and Asset Returns'. Journal of Finance, 49, 123-152.

Christiano, Lawrence J. (1987). 'Cagan's Model of Hyperinflation under Rational Expectations'. International Economic Review, (February) 28: 33-49.

Christiano, L.J., M. Eichenbaum, and D. Marshall (1991). 'The Permanent Income Model Revisited'. Econometrica, 59: 397-423.

Cochrane, J.H. (1989). 'The Sensitivity of Tests of the Intertemporal Allocation of Consumption to Near-Rational Alternatives'. American Economic Review, 79: 319-337.

Cochrane, J. and L.P. Hansen (1992). 'Asset Pricing Explorations for Macroeconomics'. NBER Macroeconomics Annual 1992, Cambridge, MA: The MIT Press, 115-165.

Constantinides, G.M. (1990). 'Habit Formation: A Resolution of the Equity Premium Puzzle'. Journal of Political Economy, 98, 519-43.

Deaton, Angus (1991). 'Saving and Liquidity Constraints'. Econometrica, Vol. 59 (5):1221-48.

Descartes, Rene (1901). The Method, Meditations, and Philosophy of Descartes. Translated by John Vietch, M. Walter Dunner, Washington and London. 
Epstein, L.G. (1988). 'Risk Aversion and Asset Prices'. Journal of Monetary Economics, Vol. 22, No. 2, September; pp. 179-192.

Epstein, L.G. and T. Wang (1994). 'Intertemporal Asset Pricing Under Knightian Uncertainty'. Econometrica, 62:3, 283-322.

Epstein, L.G., and S.E. Zin (1989). 'Substitution, Risk Aversion, and the Temporal Behavior of Consumption and Asset Returns: A Theoretical Framework'. Econometrica, 57:4, 937-969.

Epstein, L.G., and S.E. Zin (1990). 'First-Order Risk Aversion and the Equity Premium Puzzle'. Journal of Monetary Economics, 26:3, 387-407.

Frances, B.A. (1987). A Course in H-infinity Control Theory. Lecture Notes in Control and Information Sciences, vol. 88.

Gallant, R., L.P. Hansen, and G. Tauchen (1990). 'Using Conditional Moments of Asset Payoffs to Infer the Volatility of Intertemporal Marginal Rates of Substitution'. Journal of Econometrics, 45: 145-179.

Gilboa, I. and D. Schmeidler (1989). 'Maxmin Expected Utility with Non-unique Prior'. Journal of Mathematical Economics, 18, 141-153.

Glover, K., and J.C. Doyle (1988). 'State-space Formulae for All Stabilizing Controllers that Satisfy an $H_{\infty}$-norm Bound and Relations to Risk Sensitivity'. Systems Control Letters, 11, 167-172.

Hall, R.E. (1978). 'Stochastic Implications of the life cycle-Permanent Income Hypothesis: Theory and Evidence'. Journal of Political Economy, 91, 249-265.

Hansen, L.P. (1987). 'Calculating Asset Prices in Three Example Economies'. In T.F. Bewley (ed.), Advances in Econometrics, Fifth World Congress Vol. 1, Cambridge University Press, New York.

Hansen, L.P., J. Heaton, E.G.J. Luttmer (1995). 'Ecnometric Evluation of Asset Pricing' Models'. The Review of Financial Studies, 8:2, 237-274.

Hansen, L.P. and R. Jagananthan (1991). 'Implications of Security Market Data for Models of Dynamic Economies'. Journal of Political Economy, 99:225-262.

Hansen, L.P., W. Roberds and T.J. Sargent (1991). 'Time Series Implications of PresentValue Budget Balance and of Martingale odels of Consumption and Taxes'. Rational Expectations Econometrics, Westview Press, 121-161.

Hansen, Lars Peter and Thomas J. Sargent (1983). 'Aggregation over Time and the Inverse Optimal Predictor Problem for Adaptive Expectations in Continuous Time'. International Economic Review, (February) 24: 1-20.

Hansen, L. P., and T. J. Sargent (1993). 'Seasonality and Approximation Errors in Rational Expectations Models'. Journal of Econometrics, 55 , 21-55.

Hansen, L.P., and T.J. Sargent (1995). 'Discounted Linear Exponential Quadratic Gaussian Control'. IEEE Transactions on Automatic Control, 40:5, 1995.

Hansen, L.P., and T.J. Sargent (1996). Recursive Linear Models of Dynamic Economies. forthcoming. 
Harrison, J.M., and D.M. Kreps (1979). 'Martingales and Arbitrage in multiperiod Securities Markets'. Journal of Economic Theory, 20, 381-408.

Heaton, John (1993). 'The Interaction Between Time-Nonseparable Preferences and TimeAggregation'. Econometrica, 61:2, 353-385.

Heaton, J. (1995). 'An Empirical Investigation of Asset Pricing with Temporally Dependent Preference Specifications'. Econometrica, Vol. pages 681-718.

Jacobson, David H. (1973). 'Optimal Stochastic Linear Systems with Exponential Performance Criteria and Their Relation to Deterministic Differential Games'. IEEE Transactions on Automatic Control, Vol AC-18, No. 2, April 1973, 124-131.

Jacobson, David H. (1977). Extensions of Linear-Quadratic Control, Optimization and Matrix Theory. Academic Press, New York.

James, M.R.. (1992). 'Asymptotic Analysis of Nonlinear Stochastic Risk-Sensitive Control and Differential Games'. Math. Control, Signals, and Systems, 5, 401-417.

James, M.R.. 'Recent Developments in Nonlinear $H_{\infty}$ Control'. , .

Kandel, S., and R.F. Stambaugh (1991). 'Asset Returns and Intertemporal Preferences'. Journal of Monetary Economics, 27:1, 40-71.

Kreps, D.M., and E.L. Porteus (1978). 'Temporal Resolution of Uncertainty and Dynamic Choice Theory'. Econometrica, 46, 185-200.

Krusell, Per and Tony Smith (1996). 'Rules of Thumb in Macroeconomic Equilibrium: A Quantitative Analysis'. Journal of Economic Dynamics and Control, 20: 527-558.

Leland, Hayne (1968). 'Savings and Uncertainty: the Precautionary Demand for Savings'. Quarterly Journal of Economics, Vol. LXXXII, August, pp. 465-473.

Ljung, L., (1978). 'Convergence analysis of Parametric Identification Methods'. IEEE Transactions on Automatic Control, 23, 770-783.

Lucas, R. E., Jr. (1975). 'An Equilibrium Model of the Business Cycle'. Journal of Political Economy, 83, 1113-1144.

Lucas, R.E., Jr. (1978). 'Asset prices in an Exchange Economy'. Econometrica, 46, $1429-1445$.

Marcet, Albert and Thomas J. Sargent (1989). 'Convergence of Least Squares Learning' Mechanisms in Self-Referential Linear Stocahstic Models'. Journal of Economic Theory, 48: pp. 337-368.

Mehra, R. and E.C. Prescott (1985). 'The Equity Premium Puzzle: A Solution?'. Journal of Monetary Economics, 22:133-136.

Melino, Angelo and Larry G. Epstein (1995). "An Empirical Analysis of Asset Returns uner "Non-Bayesian Rational Expectations". Mimeo. University of Toronto, September.

Miller, B.L., (1974). 'Optimal Consumption with a Stochastic Income Stream'. Econometrica, 42, 253-266.

Muth, John F. (1960). 'Optimal Properties of Exponentially Weighted Forecasts'. J. American Statistics Association, (June) 55: 299-306.

Obstfeld, M. (1994). 'Evaluating Risky Consumption Paths: The Role of Intertemporal 
Substitutability'. European Economic Review, 38, 1471-1486.

Prescott, E. C., (1986). 'Theory Ahead of Business Cycle Measurement'. In K. Brunner and A. H. Meltzer (eds.), Real Business Cycles, Real Exchange Rates and Actual Policies. ,. Carnegie-Rochester Conference Series on Public Policy 25, 11-14

Ryder, H.E., Jr. and G.M. Heal (1973). 'Optimal Growth with Intertemporally Dependent Preferences'. R.E. Stud, 40: pp. .

Sundaresan, M. (1989). 'Intertemporally Dependent Preferences and the Volatility of Consumption and Wealth'. Review of Financial Studies, 2, 73-89.

Tallarini, T. D. (1996). 'Risk Sensitive Real Business Cycles'. Mimeo. Carnegie-Mellon University.

Van Der Ploeg, Frederick (1993). 'A Closed-form Solution for a Model of Precautionary Saving'. Review of Economic Studies, 60, 385-395.

Weil, P. (1989). 'The Equity Premium Puzzle and the Riskfree Rate Puzzle'. Journal of Monetary Economics, 24, 401-421.

Weil, P. (1990). 'Nonexpected Utility in Macroeconomics'. Quarterly Journal of Economics, 105, 29-42.

Weil, P. (1993). 'Precautionary Savings and the Permanent Income Hypothesis'. Review of Economic Studies, 60, 367-383.

Whittle, P. (1982). Optimization Over Time. Volume 1, Chichester: John Wiley \& Sons. White, H. (1982). 'Maximum likelihood estimation of misspecified models'. Econometrica, $50,1-26$.

Whittle, P. (1983). Optimization Over Time. Volume 2, Chichester: John Wiley \& Sons.

Whittle, P. (1989). 'Risk-sensitive Linear Quadratic Gaussian Control'. Adv. Appl. Prob, 13, 764-777.

Whittle, P. (1990). Risk-sensitive Optimal Control. John Wiley \& Sons, New York.

Zames, G. (1981). 'Feedback and Optimal Sensitivity: Model Reference Transformations, Multiplicative Seminorms, and Approximate Inverses'. IEEE Trans. Auto Control, vol. AC-26, 301-320.

Zeldes, Stephen P. (1989). 'Optimal Consumption with Stochastic Income: Deviation from Certainty Equivalence'. Quarterly Journal of Economics, May, Vol. CIV, pp. 275-298.

Zhou, K., J.C. Doyle and K. Glover (1996). Robust and Optimal Control. Upper Saddle River, NJ: Prentice-Hall, Inc. 